\title{
DENSE FUNCTIONAL AND MOLECULAR READOUT OF A CIRCUIT HUB IN SENSORY CORTEX
}

Cameron Condylis ${ }^{1,2}$, Abed Ghanbari ${ }^{3}$, Nikita Manjrekar ${ }^{3}$, Karina Bistrong $^{3}$, Shenqin Yao ${ }^{4}$,

5 Zizhen $\mathrm{Yao}^{4}$, Thuc Nghi Nguyen ${ }^{4}$, Hongkui Zeng ${ }^{4}$, Bosiljka Tasic ${ }^{4}$, Jerry L. Chen ${ }^{1,2,3,5^{*}}$

${ }^{1}$ Department of Biomedical Engineering, Boston University, Boston MA 02215, USA

${ }^{2}$ Center for Neurophotonics, Boston University, Boston MA 02215, USA.

${ }^{3}$ Department of Biology, Boston University, Boston MA 02215, USA.

${ }^{4}$ Allen Institute for Brain Science, Seattle, WA 98109, USA.

${ }^{5}$ Center for Systems Neuroscience, Boston University, Boston MA 02215, USA.

*corresponding author

\section{ABSTRACT}

Information processing in the neocortex is carried out by neuronal circuits composed of different cell types. Recent census of the neocortex using single cell transcriptomic profiling has uncovered more than 100 putative cell types which subdivide major classes of excitatory and inhibitory neurons into distinct subclasses. The extent to which this molecular classification predicts distinct functional roles during behavior is unclear. Here, we combined population recordings using twophoton calcium imaging with spatial transcriptomics using multiplexed fluorescent in situ hybridization to achieve dense functional and molecular readout of cortical circuits during behavior. We characterized task-related responses across major transcriptomic neuronal subclasses and types in layer $2 / 3$ of primary somatosensory cortex as mice performed a tactile working memory task. We find that as neurons are segregated into increasingly discrete molecular types, their task-related properties continue to differentiate. We identify an excitatory cell type, Bazla, that is highly driven by tactile stimuli. Bazla neurons homeostatically maintain stimulus responsiveness during altered sensory experience and show persistent enrichment of subsets of immediately early genes including Fos. Measurements of functional and anatomical connectivity reveal that upper layer 2/3 Bazla neurons preferentially innervate somatostatin-expressing inhibitory neurons. We propose that this connection motif reflects a sensory-driven circuit hub that orchestrates local sensory processing in superficial layers of the neocortex. 


\section{MAIN TEXT}

\section{Introduction}

Cells of the neocortex can be defined based on their molecular composition, the diversity of which is reflected in their transcriptome. The transcriptional profiles observed across this brain region indicate that cortical populations can be hierarchically subdivided into multiple putative transcriptomic cell classes (e.g., GABAergic, glutamatergic), subclasses (e.g., GABAergic Pvalb) and types (e.g., GABAergic Pvalb Vipr2) ${ }^{1,2}$. Even within a single layer of one cortical area, transcriptional diversity remains high ${ }^{3}$. Evidence suggests that this organization may have developmental origins ${ }^{4,5}$, reflect anatomical specificity ${ }^{6,7}$, or physiological properties ${ }^{8,9}$. The extent to which this diversity relates to information encoding during goal-directed behavior is unclear.

The ability to link molecularly-identified neurons with their function during behavior requires monitoring the activity of cell types in vivo. Traditional approaches to label cell types using transgenic lines or post-hoc immunohistochemistry are limited to 1-3 molecular markers ${ }^{10,11}$. This has restricted investigations to classes of excitatory and inhibitory neurons at the broadest hierarchical levels of cell type diversity. Recently-developed techniques for multiplexed spatial transcriptomics dramatically increase the number of genes that can be simultaneously identified in tissue $^{12-16}$. Combinatorial expression patterns of multiple genes can then be used to define finer divisions in the transcriptomic taxonomy corresponding to more specific neuronal subclasses and types. Further, spatial profiling of gene expression in intact tissue readily enables dense multimodal registration of anatomical and functional measurements across neurons within a single sample ${ }^{17}$. In order to 'crack' the neuronal circuits underlying behavior, we developed a platform

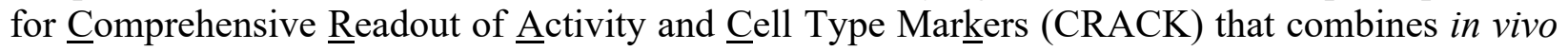
two photon calcium imaging with post-hoc multiplexed fluorescent in situ hybridization. We applied the CRACK platform to study the function of newly identified cell types in layer 2/3 (L2/3) of primary somatosensory cortex (S1).

\section{CRACK platform}

The CRACK platform employs a multi-area two-photon microscope ${ }^{18}$ configured to perform simultaneous population calcium imaging across multiple tissue depths, providing 3D spatial information of neuron location for later post-hoc identification (Fig. 1a, Supplementary Fig. 1, Supplementary Video 1). Following functional in vivo experiments, tissue encompassing the imaged volume is sectioned parallel to the imaging plane. The tissue is embedded in hydrogel and cleared ${ }^{19}$ in order to facilitate labeling of mRNA transcripts using hybridization chain reaction fluorescence in situ hybridization (HCR-FISH) ${ }^{20}$ and confocal imaging. Since HCR-FISH is a DNA-based labeling strategy, probes for different mRNA transcripts are labeled, imaged, and then stripped using DNAse across multiple rounds. In order to re-identify and register in vivo neurons across multiple rounds of HCR-FISH, we dedicate one imaging channel (561) to repeated labeling and imaging of transcripts of the red genetically encoded calcium indicator, RCaMP1.07, used for functional imaging ${ }^{21}$ (Supplementary Fig. 2, Supplementary Note S.1). Other imaging channels are used for labeling cell type-specific markers (Supplementary Table 1).

While small numbers of genes can be read out through multiple rounds of sequential staining, a barcode readout scheme provides high read depth (100-1000 genes) in an error robust manner. Using barcode readouts to decode arbitrary gene sets relies on single-molecule mRNA resolution which is sensitive to image registration errors and has only been demonstrated in thin tissue sections $(<40 \mu \mathrm{m})^{12,16}$. To obviate the need for single-molecule mRNA-resolution 
registration so that larger volumes of tissue $(150-300 \mu \mathrm{m})$ can be imaged and analyzed, we programmed our barcode for cellular-resolution readout. This approach relies on prior knowledge of expression patterns to select genes with non-overlapping expression at the cellular level for each imaging channel and hybridization round. Through this, binary decoding at each round occurs at the cellular level rather than the mRNA level. This approach is highly compatible with identifying molecular cell types that are defined by non-overlapping gene expression patterns.

To characterize the hierarchical organization of cell type diversity in L2/3 of S1, we analyzed single cell RNA sequencing (scRNAseq) data from S1 acquired as part of a larger study of the molecular diversity of the isocortex ${ }^{22}$. In this survey, L2/3 pyramidal neurons in S1 were observed to be segregated into three molecular cell types (Adamts2, Bazla, and Agmat) based on combinatorial expression patterns (Supplementary Fig. 3). Excitatory neurons in L2/3 show both cell-type-specific and area-specific gene expression patterns. When comparing S1 cells types to those in primary visual (V1) and anterior lateral motor (ALM) cortex, Bazla and Agmat cells showed similarity to L2/3 cell types identified in V1 and ALM, whereas Adamts2 cells were present in V1 but not ALM ${ }^{1}$. Molecularly-defined excitatory cell types have been associated with distinct laminar expression patterns or inter-area projection targets ${ }^{3,4}$. We examined whether cell type markers potentially correspond to non-overlapping populations of S1 neurons that project to secondary somatosensory cortex or primary motor cortex ${ }^{23}$. HCR-FISH of retrograde labeled L2/3 neurons indicate that the molecular identities of these transcriptomic cell types do not necessarily correspond to these two projection classes (Supplementary Fig. 4).

Inhibitory neuron cell types in S1 were shared with other cortical areas and found to be hierarchically organized ${ }^{22}$. While the major non-overlapping inhibitory subclasses (Lamp5, Pvalb, Sst, Vip) have each been investigated at the broadest level ${ }^{24,25}$, further divisions of these subclasses have not been investigated during task behavior. Thus, we sought to select gene markers that could define the next level of transcriptional subdivision (Supplementary Fig. 5). Lamp5 neurons were subdivided into two additional subclasses based on co-expression of either LIM homeobox 6 $(L h x G)$ or neuron derived neurotrophic factor $(N d n f)$. Pvalb neurons were subdivided based on expression of vasoactive intestinal peptide receptor 2 ( $V i p r 2)$. Sst neurons were subdivided based on expression of chondrolectin (Chodl), and Vip neurons were subdivided based on expression of parathryroid hormone-like hormone (Pthlh). From this information, we devised a barcode scheme for detection of 16 mRNA species across 6 rounds of staining to resolve 11 cell subclasses or types (3 excitatory, 8 inhibitory) for functional characterization (Fig. 1b-e).

\section{Task encoding across excitatory types}

To identify functional differences between transcriptomically-defined cell populations in L2/3 of S1, we performed two-photon calcium imaging on expert wild type mice $(n=7)$ performing a head-fixed whisker-based delayed non-match to sample (DNMS) $\operatorname{task}^{26}$ (Supplementary Fig. 6). In this context-dependent sensory processing task, a motorized rotor is used to deflect multiple whiskers in either an anterior or posterior direction during an initial 'sample' and a later 'test' period, separated by a 2 s delay (Fig. 2a). During the delay period and the inter-trial interval, the rotor was withdrawn to prevent whisker-rotor contact. Behavior was reported as 'go/no-go,' in which animals licked on 'go' trials for a water reward ('hit') when the presented sample and test stimulus were non-matching and withheld licking on 'no go' trials ('correct rejection') when the presented sample and test stimulus were matching. Misses on go trials were not rewarded, and false alarms on no-go trials were punished with an air puff and a time-out period. In addition to 
two-photon calcium imaging, high-speed videography was performed to monitor whisking behavior.

We previously reported diverse task-related responses in L2/3 of S1 during the DNMS $\operatorname{task}^{26}$. To characterize task-related responses for each recorded cell in a more comprehensive manner, we fit a generalized linear model (GLM) to each neuron's estimated spiking activity against a range of 'task variables' ${ }^{27}$ (Fig. 2b, Supplementary Fig. 8-9, Supplementary Note S.2). Task variables representing a related feature were grouped into 'task factors'(i.e. stimulus direction, trial category, etc.). The ability for a neuron to encode a particular task factor was determined by calculating the difference in the Akaike Information Criterion ( $\triangle \mathrm{AIC}$ ) between a full model and a partial model excluding task variables representing that task factor. A positive $\triangle \mathrm{AIC}$ value indicates reduced fit quality from the full to the partial model, revealing that the excluded task factor in the partial model is an important contributor to the modeled neuron's activity. Thus, we interpret significant, positive $\triangle \mathrm{AIC}$ values to indicate neuronal encoding of the excluded task factor (Fig. 2c, Supplementary Fig. 10, Supplementary Note S.3). We analyzed 10 task-related factors. Six of the ten task factors were defined by trial type information. This included information related to the direction of the task stimulus (direction), trial category defined by the combination of the sample and test stimulus (category), and the animal's choice during the test (choice TEST) and report period (choiceREPORT). While our previous study found no evidence of sustained activity in S1 during the delay period, we included task factors representing the sample stimulus at later points in the trial (sample encoded early in the delay period, sampleEARLY DELAY; Sample information late in the delay period, samplelate DELAY). Another set of task factors describing whisker movement and tactile-object interactions were derived from video analysis of whisker tracking and included whisker-object touch onset (touch onset), whisker-object touch offset (touch offset), and whisker kinematics (kinematics). A final task factor was derived from the activity of all other simultaneously recorded neurons to assess the level of coupling the neuron had with overall network activity (coupling) ${ }^{28}$.

We first compared differences in task encoding across the three excitatory cell types. We observed that Bazla showed the best overall GLM fit (Fig. 2d). In particular, Bazla neurons more strongly encoded whisker kinematics compared to the other two excitatory cell types $(P<0.005$, Mann Whitney $U$ test; Fig. 2f). In contrast, Adamts2 neurons more weakly encoded stimulus direction and touch offset (direction: $P<0.05$; touch offset: $P<0.02$, Mann Whitney $U$ test; Fig. 2e, Supplementary Fig. 11, ) while Agmat neurons more strongly encoded choicerePORT $(P<0.05$, Mann Whitney $U$ test). Bazla neurons also show overall higher firing rates $(P<0.005$, one-tailed Student's $t$-test) and response reliability to sample and test stimuli (Fig. 2g, Supplementary Fig. 12). Taken together, these findings suggest that Bazla neurons represent a highly active, sensorydriven L2/3 excitatory cell type.

\section{Persistent stimulus activity and Fos expression in Baz1a neurons}

Highly active, sensory-driven L2/3 S1 neurons have been previously observed to exhibit high expression of the immediate early gene, $F_{O S}{ }^{29,30}$. Single-cell RNAseq analysis in naïve, untrained mice shows that while all cell types express some number of immediate early genes (IEGs), Bazla neurons show consistent enrichment of Fos along with other subsets of IEGs (Fig. 3a). Previous studies observed Fos expression to be dynamic and driven by experience-dependent plasticity ${ }^{31}$. However, due its conserved and elevated expression in Bazla cells across naïve animals, we speculated that Fos and other IEGs may be stably expressed in Bazla neurons. To confirm this and address how it relates to neuronal function, we extended the CRACK platform to track Fos 
expression and stimulus activity during altered sensory experience using transgenic fosGFP mice ${ }^{32}$ along with virally co-expressed Rcamp 1.07 in $\mathrm{S} 1$ ( $n=3$; Fig. 3b,c). Ex vivo HCR-FISH confirmed that high fosGFP fluorescence corresponded with higher Fos mRNA and that GFP mRNA was also enriched in Bazla neurons (Fig. 3d, Supplementary Fig. 13).

FosGFP and sensory-evoked calcium responses were tracked before and after 5 days of bilateral whisker deprivation (BD). During BD, the principal whisker corresponding to the imaged $\mathrm{S} 1$ barrel column was trimmed to a minimum length so that stimulus-evoked activity could still be tracked. Overall, BD resulted in a decrease in stimulus-evoked activity after 1 day followed by a slow homeostatic compensation after 5 days similar to as previously observed ${ }^{33}(P<0.0002$, oneway ANOVA, post-hoc multiple comparison test; Fig. 3e). We first asked how fosGFP expression related to functional changes during BD. Cells were divided into 3 groups based on fosGFP expression: 1) stable low fosGFP expression across all imaging sessions; 2) stable high fosGFP expression across all imaging sessions, and; 3) dynamic fosGFP expression between at least one imaging session (Fig. 3f). All groups showed decreased stimulus-evoked activity after 1 day of BD. However after 5 days, responses in stable low and dynamic fosGFP neurons remained depressed while stable high fosGFP neurons exhibited an enhancement in sensory response magnitude compared to pre-BD conditions $(P<0.05$, Student's $t$-test).

Ex vivo cell type identification revealed that similar fractions of stable high fosGFP neurons were observed across all excitatory cell types before deprivation. However, during BD, there was increased fosGFP turnover in Adamts2 and Agmat neurons while the fraction of stable high fosGFP cells remained unchanged in Bazla neurons $\left(P<0.05, \chi^{2}\right.$ test; Fig. 3h). Functionally, all three cell types showed reduced stimulus activity after 1 day BD while only Bazla neurons showed recovery after 5 days of $\mathrm{BD}(P<0.005$, Student's $t$-test; Fig. 3g). Taken together, this demonstrates that Bazla neurons are molecularly and functionally distinct in exhibiting stable enrichment of select IEGs and maintaining stimulus responsiveness during altered sensory experience.

\section{Task encoding in inhibitory classes and subdivisions}

We next compared task encoding in three of the major classes of inhibitory neurons (Pvalb, Sst, Vip). Lamp5 neurons were excluded from analysis due to their low numbers captured in the data set (Supplementary Table 2). Overall, Pvalb neurons exhibited the weakest coding of tactilerelated features such as direction, touch onset, kinematics, as well as samplelate DELAY (Fig 4a-c, Supplementary Fig. 14). However, the high firing rates of Pvalb neurons and associated difficulties in reliably inferring spiking activity in this subclass by calcium imaging may underestimate the strength of GLM-derived task responses ${ }^{11,34}$ (Supplementary Note S.2). We therefore focused our analysis on Sst and Vip neurons.

We sought to investigate if more task-related differences emerge as inhibitory classes are further subdivided into more discrete transcriptomic subclasses or types. As a major class, Sst showed the best overall fit to the GLM $(P<0.005$, Mann-Whitney $U$ test; Fig. 4a) and strongly encoded stimulus direction $(P<0.05$, Mann-Whitney $U$ test; Fig. 4b). We asked whether stimulus direction was encoded similarly in subclasses of Sst neurons. Sst/Chodl+ neurons express nitric oxide synthase $(N o s 1)^{35}$, display long-range axonal projection patterns ${ }^{36,37}$, and are active during slow wave sleep ${ }^{38}$. We find that during the DNMS task, Sst/Chodl+ encoded direction more weakly compared to Sst/Chodl- neurons $\left(P<5 \times 10^{-5}\right.$, Mann Whitney $U$ test; Fig. 4d,f, Supplementary Fig. 15a). These results demonstrate that stimulus-driven responses are specific to subsets of Sst neurons that do not express Chodl. 
We next compared task differences between Vip/Pthlh+ and Vip/Pthlh- neurons (Fig. 4e). Vip neurons belonging to the Pthlh+ subclass co-express choline acetyltransferase (Chat) and calretinin, also known as calbindin-2 (Calb2), typically have bipolar morphologies, and preferentially target Sst neurons ${ }^{37,39-41}$. Vip neurons belonging to the Pthlh- subclass co-express synuclein gamma (Sncg) and cholecystokinin $(C c k)$, have multipolar and basket cell morphologies, and preferentially target Pvalb neurons ${ }^{37,42}$. We found that Vip/Pthlh- neurons more strongly encoded direction, sampleEARLY DELAY, and onset than Vip/Pthlh + neurons (direction: $P<0.05$; sampleearly delay: $P<0.01$; onset: $P<0.05$, Mann-Whitney $U$ test; Fig. 4g-i, Supplementary Fig. 15b). Analysis of neuronal firing with respect to touch onset at the beginning of the sample and test period neurons showed elevated firing for Vip/Pthlh + neurons preceding touch onset, which correlated with an anticipatory increase in whisking amplitude (Fig. 4j). This pre-touch activity suggested that Vip/Pthlh + neurons are driven by free whisking behavior. To disentangle movement-related from tactile-related whisker responses, we fit neuronal activity to a GLM with whisker kinematic variables using only time periods prior to touch onset during the pre-stimulus and delay period. We found that Vip/Pthlh+ neurons more strongly encoded whisker amplitude, angle, and phase task factors during free whisking periods compared to Vip/Pthlh- neurons (amplitude: $P<0.02$; angle; $P<0.001$; phase: $P<0.05$, Mann-Whitney $U$ test; Fig. 4k-m). These results indicate that the activity related to whisking behavior previously identified in Vip neurons ${ }^{41}$ is specific to Vip/Pthlh+ neurons. Overall, these findings demonstrate further segregation of functional task properties in inhibitory neurons with increasing transcriptomic subdivisions.

\section{Network interactions between major subclasses and types}

The ability to simultaneously record across all identified cell subclasses and types enables a comprehensive characterization of cell type-specific network structures that underlie coding of task information. Non-negative matrix factorization across varying ranks can be used to capture population dynamics across distinct functional subpopulations (Supplementary Note S.4). Neurons that exhibit strong population coupling with increasing ranks suggest functional relationships with multiple subpopulations. Compared to other excitatory neurons, Bazla neurons consistently showed higher coupling across increasing coupling ranks indicating that they are highly integrated into the local L2/3 network $\left(P<0.02, F_{2,6}\right.$, repeated measures ANOVA; Fig. 5a). To investigate how this coupling relates to specific cell type populations, we constructed a GLM that included all previously described task variables while subdividing the activity of simultaneously recorded neuronal activity into different "coupling factors" according to Adamts2, Bazla, Agmat, Pvalb, Sst, and Vip transcriptomic populations (Fig. 5b). For a modeled neuron, the $\triangle \mathrm{AIC}$ for each cell type coupling factor constituted a measure of "functional connectivity" between that neuron and other simultaneously recorded cell types that could reflect either positive or negative noise correlations (Supplementary Fig. 16). From these measures, a directional weighted network graph can be constructed composed of the six cell populations as nodes and functional connectivity as edges. Task-specific networks were generated by selecting for neurons with significant $\triangle \mathrm{AIC}\left(P<0.01, \chi^{2}\right.$ test $)$ for a given task factor $(\mathbf{F i g} . \mathbf{5 c})$. This allowed us to assess the degree of interactions between cell populations encoding different task factors.

We observed different network patterns across task factors. All task factor networks exhibited cell type-specific functional connectivity weights that were greater than chance (with the exception of the network containing non-coding neurons, which exhibited random connectivity) $(P<0.05$, bootstrap test; Fig 5d). Functional connectivity was strongest amongst neurons encoding category and whisker kinematics, suggesting that encoding of these task variables involves a high degree of local interactions (Supplementary Fig. 17a). 
We further investigated the structure of these networks. For each cell type node, we used the input edge strengths to determine how other cell populations influence the activity of the measured node and the output edge strength to measure how the measured node influences the activity of other cell populations (Supplementary Fig. 17b). Overall, we observed that inhibitory neurons were more likely to be influenced by network activity patterns than excitatory neurons. Sst neurons exhibited the highest input node strength across all task conditions $(P<0.05$, bootstrap test). This is in line with evidence suggesting that Sst neurons follow local network activity ${ }^{25}$. In contrast, excitatory neurons had a greater influence on other cell types with Bazla cells showing high output node strength in 7 out of the 9 task factor networks $(P<0.05$, bootstrap test $)$. This suggests that Bazla cells orchestrate a majority of local L2/3 activity patterns.

Given the differences in node strengths across task factor networks, we asked whether functional connectivity between any two cell types reflected a dynamic relationship that depended on the task factor network or represented a stable motif that is potentially intrinsic to the underlying circuitry. To address this, we measured the overall strength of each connection by calculating the mean edge weight across task factor networks. The stability of this connection was reported as the coefficient of variation of the edge weight across task factor networks (Fig. 5e). The majority of connections exhibited variability between task factor networks that were equivalent to chance levels, suggesting that the strength of those connections are dynamic and depend on the encoded task factor. However, a subset of connections (Adamts $2 \rightarrow$ Vip, Adamts $2 \rightarrow$ Sst, Agmat $\rightarrow$ Bazla, Bazla $\leftrightarrow$ Sst; output node $\rightarrow$ input node) were consistently strong and stable across task factor networks, suggesting that they represent intrinsic functional motifs between specific cell populations $(P<0.05$, permutation test). These findings suggest that processing of task information occurs within local circuits that are composed of both dynamic and fixed interactions between transcriptomic cell populations.

\section{Cell-type specific tracing confirms intrinsic functional connectivity}

The observed functional connections that persisted across task networks could be explained by cell type-specific synaptic connections. Trans-monosynaptic rabies tracing enables input mapping to specific cell types but requires genetic access for conditional infection. Since transgenic lines for the three excitatory cell types are not available, we focused on input patterns to Sst and Vip inhibitory classes. Since Bazla neurons showed stable functional connectivity with Sst neurons but not Vip neurons, we sought to compare Bazla synaptic connectivity between these two inhibitory classes. Using Sst-IRES-Cre $(n=4)$ and Vip-IRES-Cre $(n=4)$ mice $^{43}$, L2/3 Sst and Vip starter cells were first labeled using a Cre-dependent AAV expressing TVA, CVS-N2cG, and dTomato, followed by delivery of the EnvA-pseudotyped CVS-N2c $(\Delta G)$ rabies virus expressing histone-GFP ${ }^{44}$ (Fig. 6a,b). We first examined the sublaminar distribution of histone-GFP positive inputs (nGFP+) to Sst and Vip cells across L2/3. Overall, Sst and Vip neurons received greater number of inputs from cells located in deeper L2/3 $(>200 \mu \mathrm{m}$ below the pia) compared to superficial L2/3. However, we found Sst neurons received more of their inputs from superficial L2/3 neurons compared to Vip neurons (Sst: $28.9 \pm 1.6 \%$; Vip: $21.5 \pm 2.8 \%$; mean \pm s.e.m.; $P<0.02$, one-tailed Student's $t$-test; Fig. 6c). Multiplexed HCR-FISH from the CRACK platform was performed to identify cell type of nGFP+ input neurons. The overall density Bazla inputs was greater for Sst neurons compared to Vip neurons (Sst: $12.2 \pm 1.3 \%$; Vip: $8.1 \pm 1.5 \%$; $P<0.01$; onetailed Student's $t$-test; Fig. 6d). This difference was greatest among cells in superficial L2/3 (Sst: $21.1 \pm 1.7 \%$; Vip: $16.9 \pm 1.6 \% ; P<0.05$; one-tailed Student's $t$-test; Fig. 6e). These results reveal differential sublaminar organization of the input patterns to Sst and Vip neurons and identify a cell 
type specific synaptic connection between superficial L2/3 Bazla and Sst neurons that is consistent with functional connectivity measurements.

\section{Discussion}

In conclusion, we have developed a platform to densely survey the functional and molecular properties of neuronal populations in vivo and have applied it to study cell types in L2/3 of S1. Through this, we have shed light on the role of Bazla neurons in neocortical function. Enriched Fos expression suggests that Bazla neurons are members of a previously described, highly interconnected FosGFP population that operates as a network hub in $\mathrm{S}^{29}$. S1 is important for both tactile feature discrimination as well as sensorimotor integration for object localization ${ }^{45}$. Superficial L2/3 pyramidal neurons are laminarly situated to integrate both top-down motor and associative signals arriving in L1 onto apical dendrites with bottom-up sensory signal arriving from L4 onto basal dendrites ${ }^{46,47}$ (Fig. 6f). Basal dendrites also contain highly recurrent, lateral connections between neighboring excitatory neurons ${ }^{48}$. L2/3 Sst neurons predominantly target and inhibit apical dendrite activity ${ }^{49}$. In turn, Vip neurons inhibit Sst neurons to form a disinhibitory circuit motif that enables integration of top-down signals and mediates associative memory formation in L2/3 pyramidal neurons ${ }^{41,50}$. We propose that excitatory connections from superficial Bazla neurons onto Sst neurons serves to counteract this disinhibitory circuit by driving Sst neurons to inhibit top-down inputs in neighboring pyramidal neurons. This would bias synaptic integration in local L2/3 pyramidal neurons towards bottom-up and recurrent inputs. Showing high responsiveness to sensory stimuli, Bazla neurons are well poised to respond to sensory stimulation and recruit local circuits for sensory processing. Therefore, these circuit motifs operate complementary to one another, allowing S1 to shift between gating long-range feedback inputs and engaging feedforward computations.

Bazla neurons are also functionally distinct in their ability to adapt to altered sensory experience by homeostatically maintaining their response to tactile stimuli. Sensory deprivation has been shown to transiently induce changes in IEG expression resulting in experience-dependent plasticity $^{51}$. We speculate that stable expression of Fos and other select IEGs in Bazla cells may prime this cell type to respond to changes in experience through molecular mechanisms that could adjust excitatory-inhibitory balance, synaptic scaling, or intrinsic excitability. This plasticity suggests that Bazla neurons both act to preserve existing sensory representations in the face of novel experiences and to recruit local circuits for ongoing sensory processing. The presence of cell types in V1 and ALM with similar expression profiles as Bazla neurons suggest that homologous circuits may exist across neocortical areas. Overall, the findings that we have produced from the CRACK platform provide new insight into how neural computations can be embedded within the molecular logic of neocortical circuits.

Acknowledgements We thank O. Gonen, S. Kenyon, G. Shechter, N. Weston, C. Xin for software development, A. Ahrens, G. House, K. Marmon for assistance in image analysis, N. Josephs for advice in statistical analysis, M. Economo, D. Lee, B. Scott, C. Habjan for comments on the manuscript. This work was supported by grants from a NARSAD Young Investigator Grant from the Brain \& Behavior Research Foundation, the Richard and Susan Smith Family Foundation, Elizabeth and Stuart Pratt Career Development Award, the Whitehall Foundation, Harvard NeuroDiscovery Center, National Institutes of Health BRAIN Initiative Award (R01NS109965 to J.C., and U19MH114830 to H.Z.), National Institutes of Health New Innovator Award (DP2NS111134), and National Institutes of Health Ruth L. Kirschstein Predoctoral Individual National Research Service Award (F31NS111896) to C.C.. 
Authors' Contributions. C.C. and J.L.C. designed the study. C.C. performed two-photon imaging and CRACK platform experiments. C.C. and K.B. performed retrograde tracing experiments. S.Y. and C.C. performed rabies tracing experiments. C.C., A.G., N.M., K.B., and J.L.C. performed data analysis. T.N., Z.Y., and B.T. generated and analysed the single cell transcriptomic data supervised by H.Z. C.C. and J.L.C. wrote the paper.

\section{Declaration of Interests The authors declare no competing interests.}

Materials \& Correspondence. Correspondence to Jerry L. Chen, jerry@,chen-lab.org

\section{METHODS}

Mice. Experiments in this study were approved by the Institutional Animal Care and Use Committee at Boston University, approved by the Allen Institute Animal Care and Use Committee, and conform to NIH guidelines. Behavior experiments were performed using C57BL/6J mice (The Jackson Laboratory). Input mapping experiments were performed using SST-IRES-Cre and VIP-IRES-Cre mice ${ }^{43}$. Binocular deprivation experiments were performed using B6.CgTg(Fos/EGFP)1-3Brth/J mice ${ }^{32}$. All animals were 6-8 weeks of age at time of surgery. Mice used for behavior were housed individually in reverse 12 hour light cycle conditions. All handling and behavior occurred under simulated night time conditions.

Single cell RNA sequencing analysis. Single cell RNA sequencing data in this study were previously acquired ${ }^{22}$. S1 excitatory and inhibitory subclasses and cell types obtained were identified using an iterative clustering $\mathrm{R}$ package hicat (https://github.com/AllenInstitute/hicat) as previously described ${ }^{1}$. To understand the cell type and area differences of L2/3 neurons among S1, V1, and ALM, differentially expressed genes (DEG) identified between all pairs of cell types, all pairs of areas, and all pairs of cell type and area groups (fold change $>4$, FDR 0.01, and expressed in $>=40 \%$ cells in one group). The average counts per million reads mapped (CPM) values of each DEG within each group were scaled across all the groups to between 0 and 1. A DEG was categorized as cell type specific if its normalized values were greater than 0.5 in at least two areas (or one region if the cluster is specific to one area) within a given cell type, and area-specific if its scaled values were greater than 0.5 in at least two cell types within a given area. DEGs specific to both cell type and area were defined as genes with scaled value greater than 0.5 in only one area and cell type. Immediate early genes enriched in Bazla neurons were identified as those with statistically higher CPM values compared to Adamts2 and Agmat neurons.

Animal imaging preparation. For behavior and bilateral whisker deprivation experiments, the genetically encoded calcium indicator RCaMP $1.07^{21}$ was expressed by stereotaxic injection of AAV2/PHP.eb-RCaMP1.07 into $\mathrm{S} 1\left(600 \mathrm{~nL}, \sim 1 \times 10^{9} \mathrm{vg} / \mathrm{ml}\right.$ per virus $)$. L2/3 was targeted at 1.1 $\mathrm{mm}$ posterior to bregma, $3.3 \mathrm{~mm}$ lateral, $300 \mu \mathrm{m}$ below the pial surface. A $4 \mathrm{~mm}$ cranial window was implanted over S1 to obtain optical access ${ }^{52}$. A metal headpost was implanted on the skull adjacent to the window to allow for head fixation. One week after window implantation and injections, animals were handled and acclimated to head fixation. In order to identify regions of S1 that correspond to specific whiskers, functional mapping was performed using intrinsic signal imaging. 
Behavioral task. Animals were trained on a head-fixed, whisker-based delayed non-match to sample using equipment and training protocol previously described ${ }^{26}$. Whiskers were trimmed to a single row corresponding to the selected imaging region for high-speed videography. Animals were water deprived throughout training and imaging and only received water by performing the task. Their weight was monitored daily to ensure body weight did not drop below $80 \%$ of initial weight. In the task, a motorized rotor was used to deflect whiskers in either an anterior or posterior direction. During the delay period and inter-trial interval, the rotor was withdrawn to prevent whisker-rotor contact. Behavior was reported as 'go/no-go' in which animals licked on 'go' trials for a water reward ('hit') when the presented sample and test stimulus were non-matching and withheld licking on 'no-go' trials ('correct rejection') when the presented sample and test stimulus were matching. Misses on go trials were not rewarded, and false alarms on no-go trials were punished with an air puff and a time-out period.

Bilateral whisker deprivation. Awake, head-fixed animals were subjected to passive whisker deflection. Each trial had the following structure: baseline $(3 \mathrm{~s})$, rotor approach, anterior rotation and whisker deflection (1s), posterior rotation and whisker deflection (1s). For baseline activity and fosGFP measurements, neurons were imaged for two days prior to deprivation (Day -2 and 1). Whiskers were trimmed bilaterally and imaged immediately afterwards (Day 0 ). The principle whisker was trimmed to a stub $\sim 1-2 \mathrm{~mm}$ in length, so that it would not provide sensory information to the animal but could still be deflected by the rotor. Whiskers were re-trimmed every other day. During deprivation, activity measurements were acquired at Day 1 and 5 while fosGFP measurements were acquired at Day 1, 3, and 5.

Two-photon imaging. Two-photon calcium imaging in expert animals was performed with a custom-built resonant-scanning multi-area two-photon microscope with $16 \mathrm{x} / 0.8 \mathrm{NA}$ water immersion objective (Nikon) using custom-written Scope software as previously described ${ }^{53}$. A $31.25 \mathrm{MHz} 1040 \mathrm{~nm}$ fiber laser (Spark Lasers) was used to excite the red calcium indicator RCaMP1.07. The multi-area two-photon microscope was configured to perform simultaneous imaging at $32.6 \mathrm{~Hz}$ frame rate of two imaging planes were separated by $\sim 50 \mu \mathrm{m}$ in depth. Average power of each beam at the sample was $70-90 \mathrm{~mW}$. For each animal, imaging was performed across 8-10 behavioral sessions. For bilateral whisker deprivation experiments, calcium imaging was performed across 16 different fields of view (FOV) per animal for each imaging session. Each FOV was acquired for 50 trials. For imaging fosGFP expression, a dual channel high resolution 3D stacks of RCamp1.07 and GFP were taken through the calcium-imaged area using a Ti:Sapphire laser tuned to $860 \mathrm{~nm}$ (Chameleon Ultra Laser; Coherent) and resolved using 630/69nm and 520/60nm emission filters (BrightLine), respectively. Following the conclusion of all experiments, animals were anesthetized and a high resolution 3D image volume was taken through the imaged area(s) for later registration with ex vivo tissue.

Whisker tracking and analysis. High-speed videography of whisker movement was acquired at $500 \mathrm{~Hz}$ as previously described ${ }^{26,54}$. For analysis, whiskers were automatically traced ${ }^{54}$. The angle, curvature $(\kappa)$, and location of the whisker tip at each time point was extracted for all traced whiskers. Using the mean whisker angle, a Hilbert transform was applied to determine whisking amplitude, phase, setpoint, and the reconstructed whisker angle (anglereCONSTRUCTION) $)^{55}$. The position of the rotor was automatically tracked in the video using custom scripts (MATLAB). Whisker-rotor touch was scored as events in which the tip of at least one whisker came into within 
$<5$ pixel radius of the rotor face with 'touch onset' defined as the first possible whisker contact at the beginning of the sample and test period and 'touch offset' defined as the last possible whisker contact at the end of the sample and test period. Time vectors of kinematic parameters were downsampled to the imaging frame rate for further analysis. Behavioral sessions with sub-optimal imaging conditions in which whisker tracking failed in $>20 \%$ of trials were excluded from analysis.

In vivo image analysis. All image processing was performed in MATLAB as described ${ }^{26,55}$. Twophoton images were first motion corrected using a piece-wise rigid motion correction algorithm ${ }^{56}$. For behavior experiments, regions of interest (ROIs) were extracted using constrained nonnegative matrix factorization and were labeled as active neurons. Remaining neurons in the FOV were manually segmented and labeled as inactive neurons. For bilateral whisker deprivation experiments, neurons across imaging sessions were identified by first registering each session image into a global reference image using ImageJ and MATLAB. For each neuron, ROIs were manually segmented in the reference image and applied to each session image for extraction of calcium signals. For analysis of fosGFP expression, 3D volume stacks across imaging sessions were aligned by landmark-based 3D affine registration using custom software (Neurotator). Automatically segmented 3D ROIs were obtained from registered ex vivo tissue slices corresponding to in vivo imaged areas and were overlaid for each imaged neuron. The mean fluorescence intensity for GFP and RCamp1.07 was extracted for each ROI. To control for variability in imaging conditions and background fluorescence, relative GFP intensity was expressed as $\Delta \mathrm{R} / \mathrm{R}_{0}=\left(R_{R O I}-R_{\text {neuropil }}\right) / R_{\text {neuropil }}$ where $R_{R O I}$ is the ratio between GFP and RCaMP1.07 fluorescence in the ROI and $R_{R O I}$ is the ratio between GFP and RCaMP1.07 fluorescence in the surrounding neuropil. FosGFP neurons with $\Delta \mathrm{R} / \mathrm{R}_{0}>0.2$ were identified as high expressing.

Spike estimation. Calcium signals were deconvolved using an Online Active Set method to Infer Spikes (OASIS), a generalization of the pool adjacent violators algorithm (PAVA) for isotonic regression (see also Supplementary Note S.2). First, calcium signals below baseline fluorescence (bottom $10^{\text {th }}$ percentile of signal intensity) were thresholded. For each cell, a convolution kernel with exponential rise and decay time constants were determined using an autoregressive model. The convolution kernel was applied to the calcium signals to obtain an initial spike estimate $(\hat{s})$ which was then thresholded $(\hat{s}=1)$ into binary events representing an estimated spike. For measurement of photon shot noise, signal-to-noise $(v)$ was calculated as:

$$
\text { 1) } v=\frac{\operatorname{Median}_{t}\left|F_{t+1}-F_{t}\right|}{\sqrt{f_{r}}}
$$

where the median absolute difference between two subsequent time points of the fluorescence trace, $F$, is divided by the square root of the frame rate, $f_{r}{ }^{57}$.

Trans-monosynaptic rabies tracing. For input mapping experiments, trans-monosynaptic rabies tracing was performed on transgenic Sst-IRES-Cre or VIP-IRES-Cre mice. AAV1-hSyn-DIOTVA66T-dTom-CVS-N2cG virus (titer $=5.46 \times 10^{12} \mathrm{vg} / \mathrm{mL}$ ) was injected into $\mathrm{L} 2 / 3$ of $\mathrm{S} 1$ in the left hemisphere ( $1.1 \mathrm{~mm}$ posterior to bregma, $3.3 \mathrm{~mm}$ lateral, $300 \mu \mathrm{m}$ deep) via iontophoresis at $5 \mathrm{uA}$ for $5 \mathrm{~min}$. Four weeks later, $500 \mathrm{~nL}$ of EnvA-CVS-n2c(dG)-histone-EGFP virus (titer $=5 \times 10^{9}$ $\mathrm{vg} / \mathrm{mL}$ ) was injected into $\mathrm{S} 1$ using the coordinates above via nanoinject. Animals were sacrificed after 7 days. 
Retrograde tracing. Retrograde tracing was performed to identify long-range axon projection targets of different molecular types of excitatory cells. Cholera toxin subunit-b (conjugated to AlexaFluor 555 (CTB-555; 500nl, $1 \mathrm{mg} / \mathrm{ml}$; ThermoFisher Scientific) was injected into one of two major projection targets of S1 ( $n=6$ mice for each projection target): secondary somatosensory cortex $(0.7 \mathrm{~mm}$ posterior to bregma, $4.2 \mathrm{~mm}$ lateral, $300-500 \mu \mathrm{m}$ deep $)$, or primary motor cortex (1.1 $\mathrm{mm}$ anterior to bregma, $0.6 \mathrm{~mm}$ lateral, 300-500 $\mu \mathrm{m}$. Seven days after injection, brains were perfused and fixed in 4\% PFA. Coronal tissue section $(150 \mu \mathrm{m})$ were cleared and subjected to HCR-FISH. Multi-channel image stacks were acquired in L2/3 of S1 with FISH probes in 488 and 647 and CTB labelling in 561 using LSM confocal microscope. Percentage of CTB positive cells expressing each gene was manually quantified in ten randomly selected $150 \times 150 \mu \mathrm{m}^{2}$ subregions of L2/3 (ImageJ).

Ex vivo tissue preparation. At the conclusion of in vivo imaging experiments, the cranial window was removed. Several small punctures were made in tissue surround the in vivo imaged area using a glass pipette dipped in lipophilic dye (SP-DiIC18(3) (1,1'-Dioctadecyl-6,6'-Di(4-Sulfophenyl)3,3,3',3'-Tetramethylindocarbocyanine; Fisher Scientific, cat no. D7777). Punctures were made in an asymmetrical pattern such that the orientation of the slice could be determined based on their locations. A stereoscopic image was taken of the punctures relative to blood vessels. The animal was then euthanized and transcardially-perfused using $1 \mathrm{X}$ PBS followed by $4 \%$ paraformaldehyde (PFA; 32\% stock (wt/vol); Microscopy Sciences, cat. no. 15710-S). The brain was removed and further fixed in $4 \%$ PFA overnight at $4^{0} \mathrm{C}$. The following day, the brain was mounted in $1.5 \%$ agarose gel (Agarose Molecular Bio Grade (100g); IBI Scientific, cat. no. IB70040) and sliced tangentially, parallel to the imaging plane in 150-300 um sections using a vibratome (Leica VT1000S Vibratome; Leica Biosystems). Slices were cleared using PACT-CLARITY procedure previously described $^{14,19}$ For input mapping experiments, three or four $150 \mu$ m-thick coronal slices from each animal centered around the injection site were selected for further processing.

Probe set and barcode design. HCR-FISHv3.0 probe sets consist of a target sequence that binds to mRNAs of interest paired with one of three orthogonal HCR hairpin amplifiers (B1, B2, B3) conjugated to either AlexaFluor488, AlexaFluor546, AlexaFluor647, AlexaFluor750, AlexaFluor $790^{20}$ (Molecular Instruments, Inc.). Target binding sequences for transcripts were determined from sequences deposited in NCBI RefSeq (www.ncbi.nlm.nih.gov/refseq/) with the exception of RCaMP1.07 obtained from ${ }^{21}$. Order and lot numbers for all probe sets are listed (Supplementary Table 1).

For behavior experiments, a barcode scheme was implemented for gene readout based on a Hamming code similar to as previously described ${ }^{12}$. The barcode contained two readout channels (B1-647 and B2-488). Readout of one gene was assigned to only one channel and encoded in two out of five rounds of staining. For error-robust encoding, a Hamming distance of 2 was used such that at least 2 errors were required to switch from one readout to another. For cellular-resolution readout, genes were assigned to readouts such that no co-expressed genes were present within one round and channel of staining. A sixth round of staining was added to confirm the identity of excitatory neurons using Slc17a7 in B2-488 and inhibitory neurons using Gad2 in B1-647. For registration and alignment of neurons across in vivo images and multiple round of HCR-FISH, probe sets for RCaMP1.07 in B3-546 was used across all staining rounds.

For input mapping and bilateral whisker deprivation experiments, fewer genes were read out and Fos mRNA was quantified in fosGFP animals. For these reasons, sequential multiplexed 
HCR-FISH of non-overlapping probes was performed instead of using overlapping probes as in the barcode scheme. Since GFP was expressed in both sets of tissue, readout of gene expression was used performed with B2-647 and a mixture of B1-750:B1-790 (1:1). Genes identifying cell types were selected based on results from single cell RNA sequencing of mouse S1 neurons using SMART-Seq v4 and 10x Genomics Chromium platform as previously described ${ }^{22}$ (portal.brainmap.org/atlases-and-data/rnaseq).

Multiplexed hybridization chain reaction fluorescent in situ hybridization. HCR-FISH was performed with modifications to the v3.0 protocol $^{13}$. Fixed, cleared samples were incubated in 500 $\mu \mathrm{L}$ of $30 \%$ probe hybridization buffer ( $30 \%$ formamide, $5 x$ sodium chloride sodium citrate (SSC), $9 \mathrm{mM}$ citric acid, $0.1 \%$ Tween $20,50 \mu \mathrm{g} / \mathrm{mL}$ heparin, $1 \mathrm{x}$ Denhardt's solution, and 10\% low MW dextran sulfate) at $37^{\circ} \mathrm{C}$ for 30 minutes. Samples were then moved to probe solution overnight, which comprised of $500 \mathrm{ul}$ of $30 \%$ probe hybridization buffer and $2 \mu \mathrm{L}$ of initiator probes $(1 \mu \mathrm{L}$ of odd probe and $1 \mu \mathrm{L}$ of even probe, taken from $2 \mu \mathrm{M}$ probe stock solutions) at $37^{\circ} \mathrm{C}$. The next day, samples were washed four times at $37^{\circ} \mathrm{C}$ for $30 \mathrm{~min}$ in $500 \mathrm{uL}$ of the following solutions, respectively: $75 \%$ probe wash buffer $+25 \% 5 x$ SSC (Sigma Aldrich), $50 \%$ probe was buffer + $50 \% 5 x$ SSC, $25 \%$ probe wash buffer $+75 \% 5 x$ SSC, and 100\% 5x SSC. Samples were then moved to $500 \mathrm{uL}$ of amplification buffer (5x SSC $+0.1 \%$ Tween $20+10 \%$ low MW dextran sulfate) at RT on a shaker for 30 minutes. $10 \mu \mathrm{L}$ of $3 \mu \mathrm{M}$ stock hairpin amplifier solution was snap cooled by heating to $95^{\circ} \mathrm{C}$ for 90 seconds and then allowed to cool in a dark drawer for $30 \mathrm{~min}$. $10 \mu \mathrm{L}$ of both snap-cooled odd and even hairpins were added to $500 \mu \mathrm{L}$ of amplification buffer to form the final amplification solution. Samples were moved to final amplification solution and allowed to amplify at RT overnight. The following day, samples were removed from the amplification solution and washed in 5x SSC three times at RT: 30 minutes, 30 minutes, and 15 minutes. Between staining rounds, in situ probes were removed using DNAse (Dnase I recombinant; Sigma Aldrich ct. no. 04716728001) as described ${ }^{15}$. Samples were incubated for 30 min at RT in $500 \mu \mathrm{L}$ of $1 \mathrm{X}$ incubation buffer (40 mM Tris-HCl, $10 \mathrm{mM} \mathrm{NaCl}, 6 \mathrm{mM} \mathrm{MgCl2,} 1 \mathrm{mM} \mathrm{CaCl2}$ ). Samples were then moved to $500 \mu \mathrm{L}$ of $1 \mathrm{X}$ incubation buffer with Recombinant DNase I (10 U/ $\mu \mathrm{L})$ for 4 hours at RT. Samples were then washed three times for 30 minutes at RT in $500 \mu \mathrm{L}$ of MT Probe Wash Buffer. For bilateral whisker deprivation experiments, cell nuclei were stained using DAPI during the first round of HCR-FISH staining (DAPI Fluoromount-G; SouthernBiotech).

Confocal imaging. Tissue sections were mounted on $75 \mathrm{~mm}$ x $25 \mathrm{~mm}$ glass microscope slides (Fisher Scientific) with Fluoromount-G (SouthernBiotech) and 50mm x 22mm cover glass (Fisher Scientific). Images were acquired on one of two confocal systems: 1) a Nikon C2+ Si spectral laser scanning microscope (LSM) with Nikon Plan Apo $\lambda 20 \mathrm{x} / 0.8 \mathrm{NA}$, air objective, and $0.4094 \times 0.4094 \times 1 \mu \mathrm{m}^{3} \mathrm{XYZ}$ image voxel size, or 2) a Nikon Ti2-E body with Yokogawa Spinning Disk and Nikon CFI Apo LWD 40x/ 1.15NA, water immersion objective, and 0.1625x0.1625x0.4 $\mu \mathrm{m}^{3} \mathrm{XYZ}$ image voxel size. Only one system was used across multiple imaging rounds for a given tissue sample. For LSM-acquired images, a single image volume was sufficient to cover the in vivo imaging region. For spinning-disk-acquired images, multiple image tiles spanning the in vivo imaged region were acquired and later assembled using TeraStitcher ${ }^{58}$. For 'post-cleared' tissue prior to HCR-FISH staining, SP-DiIC 18 (3) and endogenous RCaMP1.07 expression was acquired using 488 and 561 imaging channel, respectively. For HCR-FISH stained tissues, 488, 561,647 , or 785 imaging channels used to visualize readout hairpins. DAPI staining was acquired using the 405 imaging channel. 
Ex vivo image analysis. In order to re-identify neurons imaged in vivo and across multiple rounds of HCR-FISH, all acquired images were registered to a common reference image volume. First, 2D frame-averaged structural images taken from the behavioral session were registered into an in vivo two-photon 3D image stack using landmark-based 2D affine transformation (MATLAB). Next, one round of HCR-FISH staining was designated as the common reference image volume. The in vivo volume including registered behavior sessions, 'post-cleared' volume, and all other HCR-FISH volumes were then registered to the reference image volume based on endogenous protein or HCR-FISH stained mRNA RCaMP1.07 expression. Landmark-based 3D thinplate registration was performed to generate a coarse alignment of the image volumes using custom software (Neurotator). This coarse alignment produced a registration accuracy of $<5 \mu \mathrm{m}$. For HCR-FISH stained volumes, thinplate registration was performed on the 561 imaging channel containing RCaMP1.07 expression was then applied to the remaining image channels. (Supplementary Fig. 1). For input mapping experiments, HCR-FISH stained volume registration was performed on the 488 imaging channel containing GFP expression of input neurons.

Following coarse alignment, an automatic fine-scale 2D rigid alignment was applied to each z-frame of HCR-FISH stained volumes using matrix-multiply discrete Fourier transform ${ }^{59}$. Registration was performed on the 561 imaging channel for behavior and bilateral whisker deprivation experiments or the 488 channel for input mapping experiments and then applied to the remaining image channels. For LSM-acquired images, fine-scale alignment was performed on the entire image stack. For spinning-disk-acquired images, sub-volumes corresponding to regions containing individual neurons imaged in vivo were first isolated and then subjected to fine-scale alignment in order to reduce CPU processing time.

In order to characterize gene expression in identified neurons, 3D cell segmentation was performed on image volumes. For behavior experiments, segmentation was performed using RCaMP1.07 expression. Following fine alignment, image stacks of RCaMP1.07 transcript expression across all imaging rounds were merged into a single volume stack. Segmentation was performed on the merged volume stack to obtain a single 3D segmented ex vivo ROI applied to each imaging round. For LSM-acquired images, segmentation was performed on HCR-FISH stained somatic RCaMP1.07 using Modular Interactive Nuclear Segmentation ${ }^{60}$. For spinningdisk-acquired images, images stacks were pre-filtered to normalize signal intensity and to label nuclei within RCaMP1.07-expressing neurons using 3D Weka Segmentation ${ }^{61}$. The resulting filtered images were merged with the original images and then segmented using Baxter Algorithm $^{62}$. For whisker deprivation experiments, segmentation was performed on DAPI stained nuclei. The resulting ROI was expanded radially by 10 voxels to better encompass the somatic region. RCaMP1.07 fluorescence within each ROI was use determined to determine whether identified cells expressed RCaMP1.07. For input mapping experiments, segmentation was performed on nGFP expression. The resulting ROI was expanded radially by 10 voxels to better encompass the somatic region. All ex vivo segmented neurons were manually validated using Neurotator.

Segmented in vivo ROIs were matched with segmented ex vivo ROIs by a combination of point cloud registration of ROI centroids and pixel overlap. Candidate matches were identified by nearest neighbor sorting (MATLAB). Overlap was calculated as:

2) overlap $=\sqrt{\text { overlap }_{\text {invivo }}^{2}+\text { overlap }_{\text {exvivo }}{ }^{2}}$ 
where overlapinvivo is the fraction of in vivo ROI pixels overlapping with the ex vivo ROI and

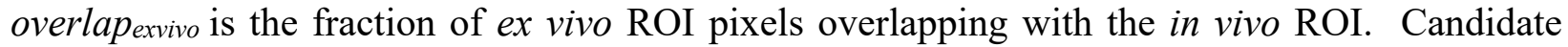
matches were rejected if overlap $<0.5$. Matching ROIs were manually validated using Neurotator. In vivo imaged ROIs determined not be contained within the ex vivo tissue volume were assigned to be unlabelled (UNL) neurons and excluded from cell type identification.

For behavior experiments, binary readout of gene expression in each HCR-FISH imaging round was manually scored by visual inspection using Neurotator. For decoding, barcodes were designed to avoid overlapping gene expression for each round of staining. However, cells that coexpress genes assigned to the same imaging channel can occasionally produce ambiguity in decoding. To resolve these situations, a combinatorial list of candidate barcodes was listed based on the barcode scheme used in the experiment and all possible gene expression patterns. From this list, non-unique barcodes were excluded as well as barcodes in which exhibitory and inhibitory markers were co-expressed. Co-expressed genes were identified from this plausible list of barcodes. Any cells with ambiguous barcodes were assigned as UNL neurons and excluded from cell type identification. While the use of a Hamming code enables error correction of the barcode readout, no error correction was performed.

For input mapping and bilateral whisker deprivation experiment, the Python library starFISH was implemented for single mRNA spot detection and quantification ${ }^{63}$. Spots were detected using min_sigma $=0.5$, max_sigma $=7$, and num_sigma $=15$. Spot detection threshold was set using Otsu's method from the scikit-image library. Spot detection performance was verified visually. The number of spots were quantified within each ROI and expression levels were expressed as the number of spots per $\mu \mathrm{m}^{3}$. For each round of staining, background spot density was quantified from the surrounding neuropil across tissue subvolumes and binary readout was determined if the ROI spot density exceeded 2 times the $95^{\text {th }}$ percentile of background spot density.

Cell types were identified using gene expression patterns. While inhibitory classes and subclasses are identified by largely non-overlapping genes, excitatory cell types were identified by combinatorial expression patterns. Specifically, Adamts2 neurons were identified by coexpression of $F s t$ and $N g b$. Balza neurons were defined by expression of either Penk or Rrad and co-expression of either Fst, $\mathrm{Ngb}, \mathrm{S100a6}$, or Coch. Agmat neurons were identified by expression of either S100a6 or Coch but no co-expression of Fst, Ngb, Rrad, or Penk. Neurons not conforming to these expression patterns were assigned as UNL neurons.

For input mapping experiments, the density of input neurons along L2/3 was calculated as the number of nGFP+ cells within a $50 \mu \mathrm{m}$ sliding window along laminar depth divided by the total number of nGFP+ cells within L2/3 for each imaged coronal slice.

Analysis of stimulus- and behavior-related activity. To assess the link between neuronal responses and various explanatory variables, a Poisson GLM was fit to each neuron's deconvolved spike train across a recording $\operatorname{session}^{27}$ (see also Supplementary Note S.3). The model defines probability in terms of a time-varying spike rate, $\lambda_{t}$, given by:

3) $\lambda_{t}=\exp \left(\sum_{i} w_{i} x_{i}(t)\right)$ 
where $x_{i}(t)$ represents the time course for the $i$ th explanatory variable, and $w_{i}$ represents the effect of this variable on the neuron's probability of spiking ${ }^{64}$. The regularized log-likelihood of a spike train for an individual trial, assuming a Poisson distribution, is given by:

$$
\text { 4) } \log p\left(r \mid x, \theta_{w}\right)=\sum_{t=0}^{T} r_{t} \log \lambda_{t}-\Delta \sum_{t=0}^{T} \lambda_{t}+\gamma\|w\|+C
$$

where $\Delta$ is the time bin size, $\mathrm{T}$ is the number of time bins in the trial, $r_{t}$ is the spike count at time $t$, and $\gamma$ is the lasso penalty term which encourages sparseness on the weights, $w$. All GLMs were fit using MATLAB's lassoglm function with a Poisson link function, 6 penalty values $(\gamma)$, and 4 fold cross-validation.

To represent the time-course of task variables $x_{i}(t)$, boxcars were constructed which were designated as "true/ 1 " during time-periods of interest and "false/ 0 " elsewhere. Since task timing was fixed across trials, each trial could be broken up into six time-periods of fixed start time and duration: "pre", "sample", "early delay", "late delay", "test", and "report". Complementary covariates were represented as independent boxcars (ie. anterior stimulus and posterior stimulus; match and non-match; lick and no lick) to capture any asymmetry in the neuron's response. To assess if a neuron's activity could be explained by trial information occurring at multiple points in the trial, some task variables were represented in multiple time periods. For a given trial, the sample stimulus direction was represented as three boxcars: during the sample stimulus presentation period, during the early delay period, and during the late period. Trial category and choice were represented as a boxcar during both the test period and the report period.

Whisker kinematics and population activity were represented as continuous variables across the entire trial. All whisker kinematic features were z-scored and down-sampled to the imaging rate before including as a covariate. Whisker touch onset and offset was represented as a boxcar of width 5 bins $(150 \mathrm{~ms})$ centered at the touch event. The coupling covariate was calculated for each neuron by excluding the given neuron's spike train, then calculating the non-negative matrix factorization (NMF) of the matrix of spike trains of all other neurons in the session ${ }^{28}$. A NMF of rank $=1$ used when assessing encoding of task factors. For assessing coupling across varying NMF ranks, each rank was included as a covariate in the model and the coupling factor included all covariate ranks.

Related covariates were grouped together into 'task factors.' For each task factor, a partial model was constructed that excluded the covariates associated with this task factor. Any increase in deviance from the full model to the partial model therefore resulted from the exclusion of this task factor's covariates. Akaike Information Criterion (AIC) was used to compare deviance between partial models in which different number of covariates were excluded such that:

$$
\text { 5) } A I C=2 k-2 \ln (L)=2 k+\text { deviance }
$$

where $k$ is the number of model parameters, deviance $=-2 \ln (L)$, and $L$ is the model likelihood. The difference in AIC ( $\triangle \mathrm{AIC})$ between the full and partial model was calculated as:

6) $\triangle A I C=A I C_{\text {partial }}-A I C_{\text {full }}$

For analysis, three GLMs were constructed. Model 1 (task GLM) was constructed to assess task-related coding of neurons using task variables and task factors shown in Supplementary Fig. 8. Model 2 (free whisking GLM) was constructed to assess neuronal responses to free whisking. Only whisker kinematic covariates included in Model 1 were included in this model. Task factors 
were calculated for each of these covariates. The model only included time points consisting of the last $1.4 \mathrm{~s}$ prior to sample period touch onset and the last 1.1s prior to test period touch onset, consistent with periods of no whisker-rotor contact preceding stimulus presentation.

Model 3 (cell type coupling GLM) was constructed to assess coupling of activity of each major cell subclass or type and consisted of all task variables in Model 1 with the exception of the 'coupling" covariate for all simultaneously neurons. Instead, seven coupling covariates for the six main cell populations (Adamts2, Bazla, Agmat, Pvalb, Sst, Vip) and all other, unlabeled cells in the session (UNL) were included and consisted of the NMF of rank $=1$ of the spike trains for simultaneously recorded neurons sorted according to each cell subclass or type. Task factors in this model corresponded to each of the main cell type coupling covariates.

For bilateral whisker deprivation experiments, the mean firing rate to anterior and posterior whisker deflections were calculated. To account for direction selectivity, the maximum activity level to either stimulus direction was used as measure of a neuron's stimulus response (SR). The change in stimulus responsiveness during BD was expressed as $\left(S R_{p o s t}-S R_{p r e}\right) /\left(S R_{\text {post }}+S R_{\text {pre }}\right)$ where $S R_{\text {post }}$ is the stimulus response at either day 1 or day 5 and $S R_{\text {pre }}$ is the mean stimulus response across Day $-2,-1$, and 0 .

Network analysis. Network analysis was performed to analyze functional connectivity between cell types across different task conditions. Task-specific networks were constructed, composed of neurons exhibiting significant $\triangle \mathrm{AIC}\left(P<0.01, \chi^{2}\right.$ test $)$ to task factors in Model 1 (task GLM). A 'non-coding' network was composed of neurons that did not exhibit any significant coding for any the task variables. Information contained in each network was derived from results from Model 3 (cell type coupling GLM). A network contained six nodes that were each composed of neurons belonging to a given major cell population. An input node was defined as the neuron modeled in the cell type coupling GLM. An output node was defined as the cell types coupling factor explaining the activity of the modeled input neuron node. A directed network edge from the output node to the input node was defined as the $\triangle \mathrm{AIC}$ of a particular cell type coupling factor that explains the activity of the input node, averaged across all input node neurons.

Network strength was calculated as the mean edge weight for all edge weights in the network. The input node strength was calculated as the sum of weights of inward directed edges from output node to the input node divided by the number of inward directed edges. The output node strength was calculated as the sum of weights of outward directed edges from input node to the output node divided by the number of outward directed edges. To test the strength and stability of functional connections across task conditions, the overall connection strength was calculated as the mean edge weight across all task networks. The stability of the connection was calculated as the coefficient of variation of the edge weight across all task networks.

Statistical procedures. No statistical methods were used to predetermine sample size. The experiments performed were not randomized and the investigators were not blinded to allocation during experiments and outcome assessment. Statistical tests used are indicated in figure legends. Error bars on plots indicate standard error of the mean (s.e.m.) unless otherwise noted.

For statistical tests of task encoding, a $\chi^{2}$ test was performed to assess the significance of the GLM $\triangle \mathrm{AIC}$ values. A Mann-Whitney $U$ test was used to compare the strength of GLM $\triangle \mathrm{AIC}$ values between cell types. The Bonferroni-Holm method was used to correct for multiple comparisons. 
For statistical tests of bilateral whisker deprivation, a one-way ANOVA test with post-hoc multiple comparison test was used to assess change in stimulus response across the imaging sessions. A Student's $t$-test was used to assess differences in stimulus response between fosGFP neurons as well as between excitatory cell types. A $\chi^{2}$ test was used to compare fraction of stable high-expressing fosGFP cells between excitatory cell types. Standard deviation of the fraction of stable high-expressing fosGFP cells for a given cell type was obtained by bootstrapping with replacement. The fraction of stable high-expressing fosGFP cells was determined from subsets $(60 \%$ of total) of cells that were randomly sampled with replacement. This process was repeated 1,000 times and the standard deviation was determined from this distribution.

For statistical tests of network analysis, to determine whether a given node strength was significantly different from nodes within the same network or between networks, bootstrap sampling with replacement was performed on the neurons representing the network. The edge strengths and node strengths were recalculated from the resulting bootstrapped data set. This process was repeated 1,000 times to obtain $95 \%$ confidence intervals for significance tests. The original node strength was then compared to the bootstrapped node strength of the other nodes in the same network or the same node across networks for within- or across-network comparisons, respectively. To determine whether a given connection was stronger and more stable than chance, permutation tests were performed by shuffling cell type labels for each input node for each task network and recalculating the overall connection strength and stability. This process was repeated 1000 times to obtain a shuffled distribution. Connections that were both stronger and more stable than the $95^{\text {th }}$ percentile of the shuffled distribution were considered to be above chance. The Bonferroni-Holm method was used to correct for multiple comparisons.

For statistical tests of input mapping, a Student's $t$-test was used to assess sublaminar and cell type differences in input density between Vip and Sst classes.

Data and code availability. The data that support the findings of this study are available from the corresponding authors upon reasonable request. Any custom written code used for data acquisition or analysis in this study is available from the corresponding authors upon reasonable request. 


\section{FIGURES}
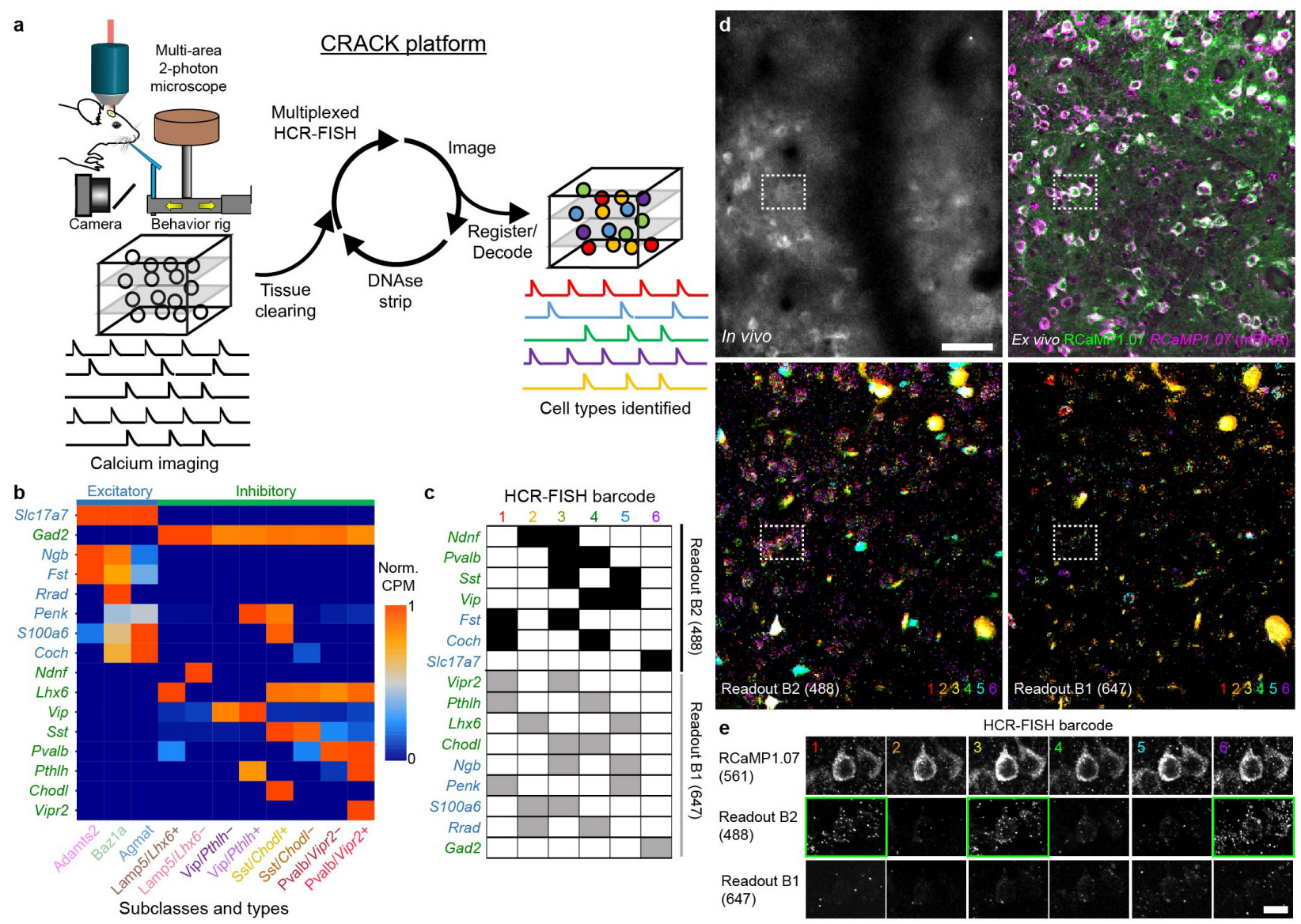

801

802

803

804

805

806

807

808

809

810

811

812

813

814

815

816

817

Figure 1. Multiplexed identification of transcriptomic cell subclasses and types in functionally imaged neurons. a, Schematic of the CRACK platform. b, Expression patterns of genes selected to identify L2/3 S1 excitatory (blue) and inhibitory (green) cell subclasses and types. c, Barcode scheme for multiplexed HCR-FISH of selected genes. One set of genes is labeled using B2-488 hairpins while another set of genes is labeled with B1-647 hairpins. d, Registration of in vivo calcium imaged neurons to ex vivo tissue section across multiple rounds of HCR-FISH. Upper left panel shows in vivo two-photon images of RCaMP1.07-expressing neurons. Upper right panel shows ex vivo confocal images of re-identified neurons showing endogeneous RCaMP1.07 fluorescence following tissue clearing (green) and following HCR-FISH of RCaMP1.07 transcripts (magenta). Overlay of B2-488 (lower left) and B1-647 (lower right) readout channels across all HCR-FISH barcode rounds. e, Decoding of in vivo imaged neuron (dotted rectangle in d) expressing Fst and Slc17a7. Individual HCR-FISH rounds are shown with RCaMP1.07 expression for registration along with B2-488 and B1-647 readout channels. Positive readouts are identified with green rectangle. Scale bars: $50 \mu \mathrm{m}, \mathbf{d} ; 20 \mu \mathrm{m}$, e. 


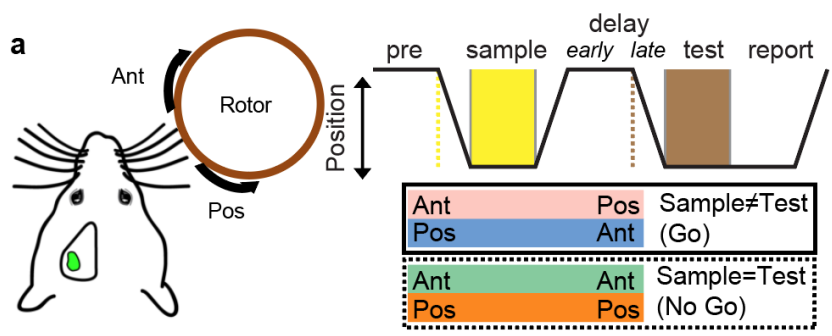

b

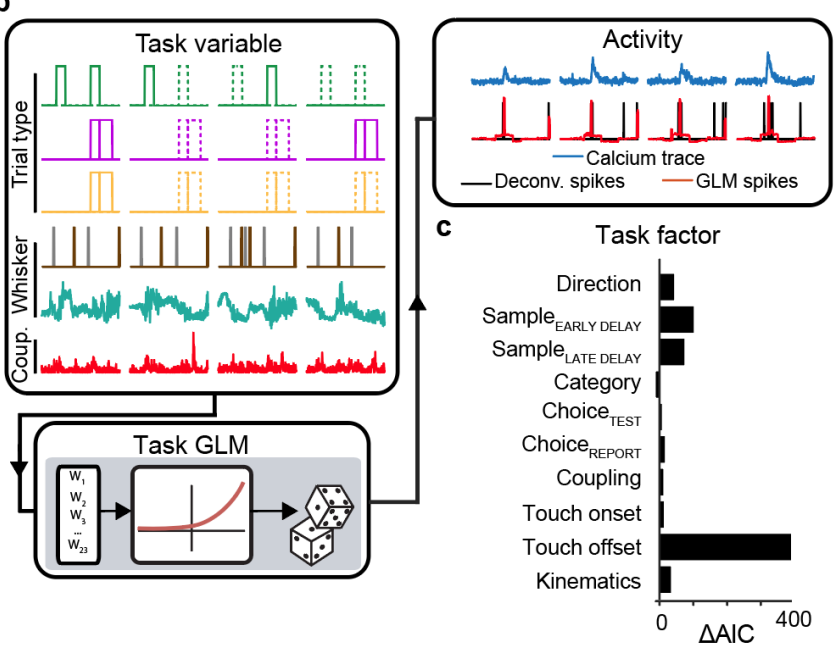

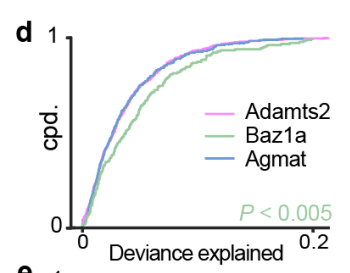
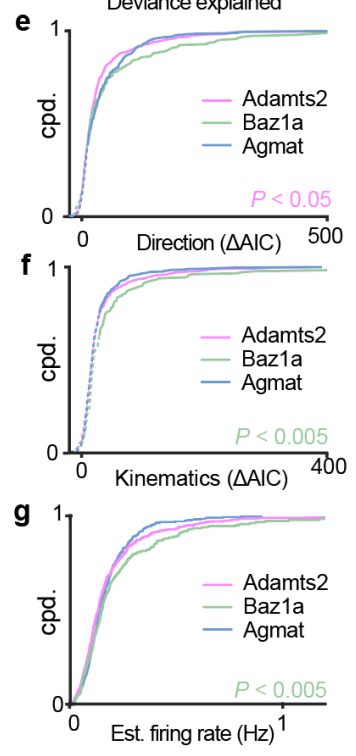

Figure 2. Task encoding across L2/3 excitatory cell types. a, Schematic of whisker-based delayed non-match to sample behavioral task. $\mathbf{b}$, Encoding of activity in individual neurons using a GLM composed of task variables including trial type information, population activity coupling, and whisker kinematics. c, Strength of groups of task variables were determined by comparing full and partial GLM fits ( $\triangle \mathrm{AIC}$ ) for individual task factors. Large, positive $\triangle \mathrm{AIC}$ indicates neuronal encoding of the individual task factor. d-g, Cumulative probability distributions of full model deviance explained (d), encoding strength of stimulus direction (e), encoding strength of whisker kinematics (f), and estimated firing rate (g) across the three excitatory cell types. (d-f, Mann Whitney $U$ test; $\mathbf{g}$, one-tailed Student's $t$-test). In e-f, solid and dotted lines correspond to significant $(P<0.01)$ and non-significant encoding strengths via $\chi^{2}$ test. $n=1107$ neurons from 7 animals. 

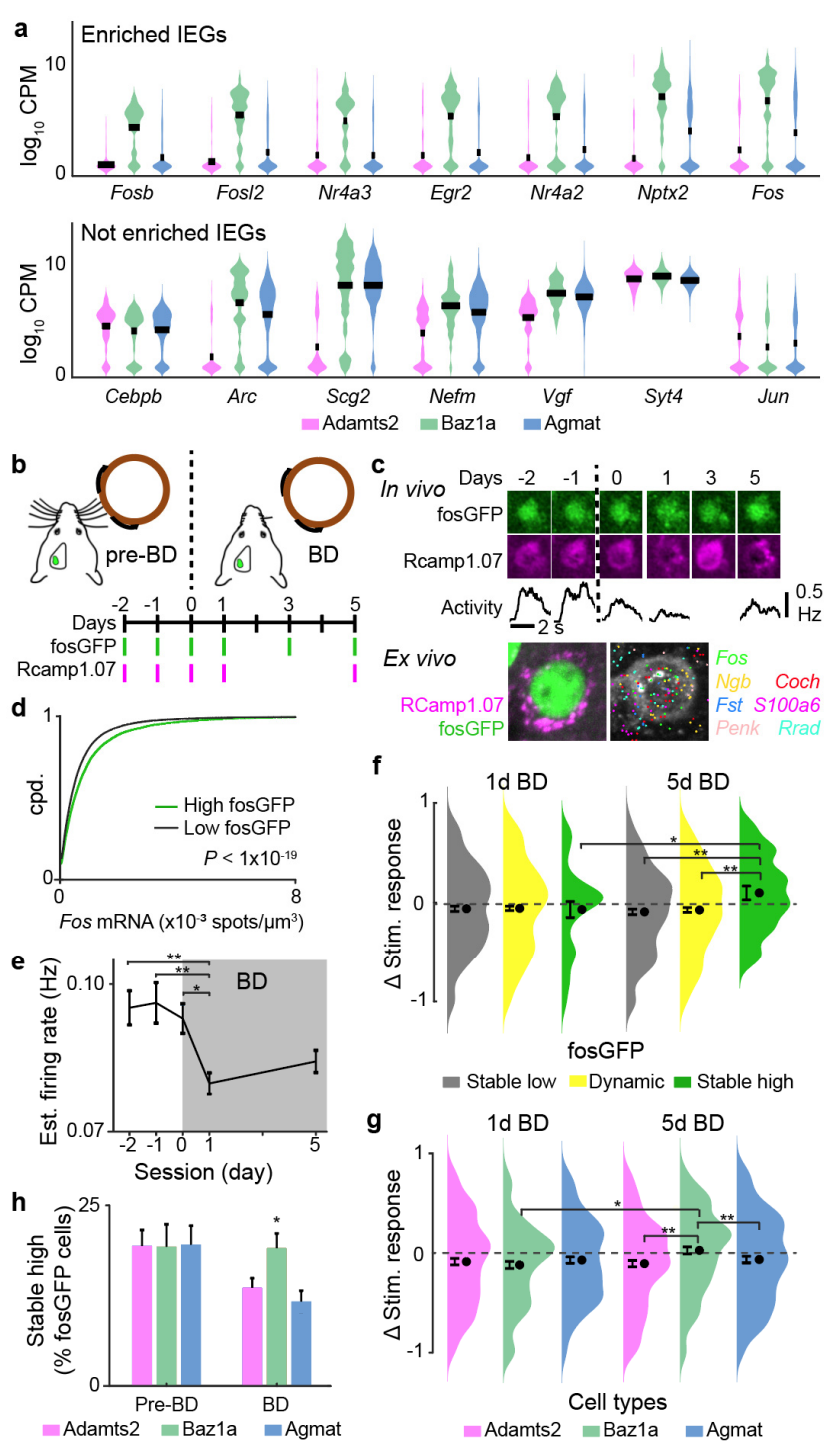

Figure 3. Persistent IEG expression and homeostatic plasticity in Baz1a neurons. a, Example of immediate early genes selectively enriched (top) and not enriched (bottom) in Bazla cells. b, Time course of bilateral whisker deprivation (BD) experiment. c, Example of Bazla neuron with stable high fosGFP expression across in vivo imaging sessions (top). Average stimulus responses during calcium imaging are shown (middle). Post-hoc identification of neuron and HCR-FISH for select genes are shown (bottom). d, HCR-FISH Fos spot density in high (1.2-fold above background) and low fluorescent fosGFP cells (two-tailed Student's $t$-test). e, Mean stimulusevoked activity before and after BD across functionally imaged neurons (one-way ANOVA with post-hoc multiple comparison test, $n=2569$ cells from 3 animals). f, Change in stimulus-evoked responses before BD versus at 1 day or 5 days BD across neurons with stable low, dynamic, and stable high fosGFP expression (two-tailed Student's $t$-test, $n=790$ cells from 3 animals). g, Change in stimulus-evoked responses before BD versus at 1 day or 5 days BD across excitatory cell types ( $\chi^{2}$ test, $n=511$ cells from 3 animals). h, Fraction of fosGFP neurons with stable high expression across all pre-BD sessions (day $-2,-1,0)$ and across all BD sessions $(1,3,5)$ for excitatory cell types (two-tailed Student's $t$-test, $n=3753$ cells from 3 animals). (* $P<0.05$, ** $P$ $<0.005$ ). Error bars = s.e.m, e-g; s.d. from bootstrap analysis, $\mathbf{g}$. 

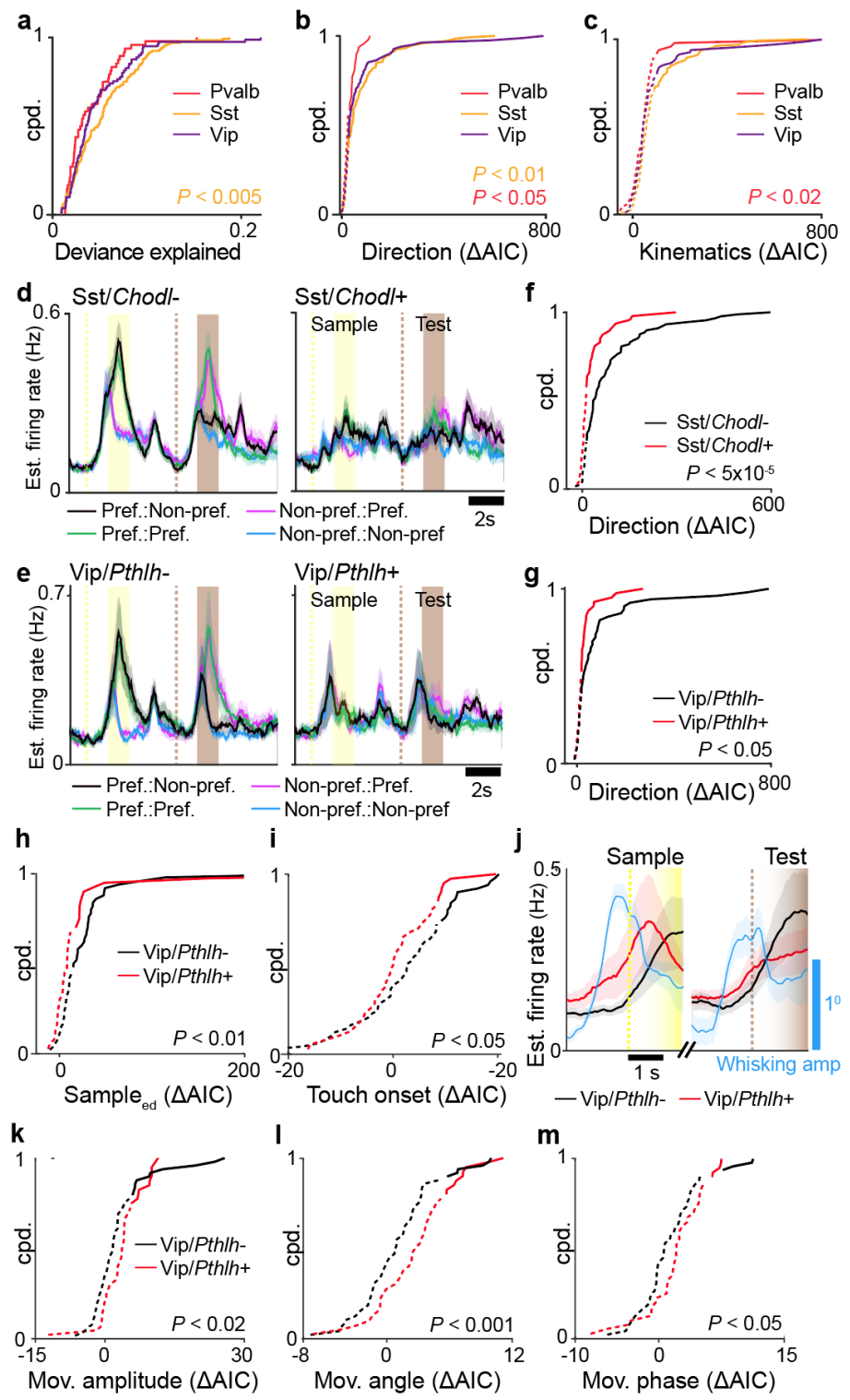

Figure 4. Task encoding across $L / 3$ inhibitory subclasses. a-c, Cumulative probability distributions for full model deviance explained (a), encoding strength of stimulus direction (b), and encoding strength of whisker kinematics (c) for three major inhibitory cell types (Mann Whitney $U$ Test). d, e, Estimated firing rate responses to preferred stimulus direction for Sst subclasses (d) and Vip subclasses (e). f, g, Cumulative probability distribution of $\triangle \mathrm{AIC}$ for task factor encoding direction for Sst subclasses (f) and Vip subclasses (g) (Mann Whitney UTest). h, i, Cumulative probability distribution of $\triangle \mathrm{AIC}$ for tasks factors encoding sampleEARLY DELAY (h) and touch onset (i) for Vip subclasses (Mann Whitney $U$ Test). j, Estimated firing rate for Vip subclasses along with mean whisking amplitude aligned to whisker-rotor touch onset preceding sample and test periods. k-m. Cumulative probability distribution of $\triangle \mathrm{AIC}$ for task factors encoding free whisking amplitude (k), angle (I) and phase (m) for Vip subclasses (Mann Whitney $U$ Test). In b-c, $\mathbf{f}-\mathbf{g}, \mathbf{m}-\mathbf{f}$, solid and dotted lines correspond to significant $(P<0.01)$ and nonsignificant encoding strengths via $\chi^{2}$ test. Shaded region in a, c corresponds to s.e.m. $n=48$ Pvalb cells, $47 \mathrm{Sst} /$ Chodl + cells, $88 \mathrm{Sst} /$ Chodl- cells, $40 \mathrm{Vip} /$ Pthlh + cells, $49 \mathrm{Vip} /$ Pthlh- cells from 7 animals. 

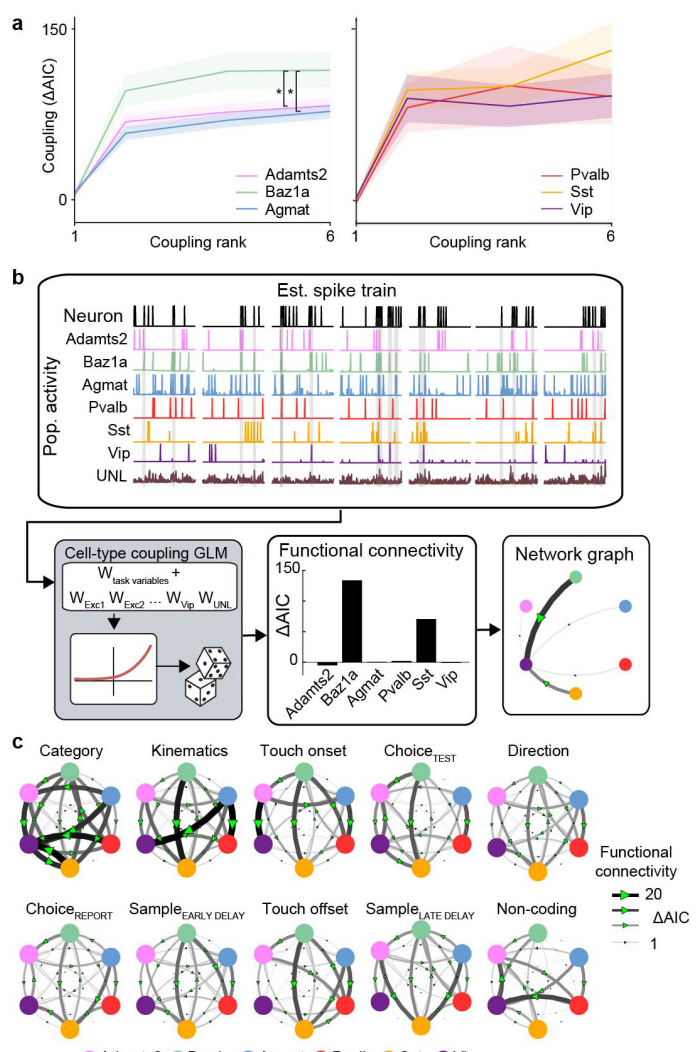

Adamts2 Baz1a Agmat Pvalb Sst $\odot$ Vip
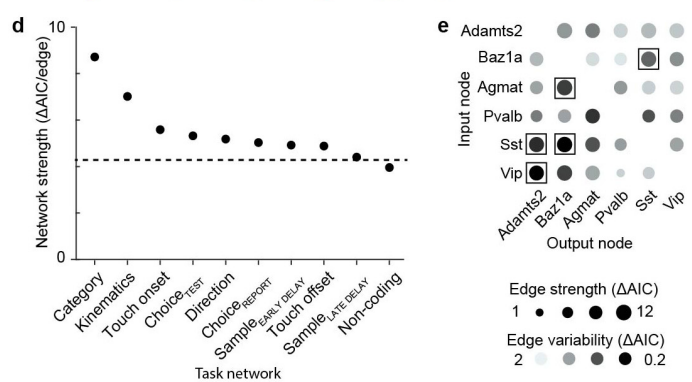

Figure 5. Cell type functional connectivity across task networks. a, Strength of coupling factor encoding across varying coupling ranks $\left({ }^{*} P<0.02\right.$, repeated measures ANOVA test, $\left.F_{2,6}\right)$. b, Schematic of network analysis for example neuron. Activity from simultaneously recorded excitatory types and inhibitory subclasses were used to construct a cell type coupling GLM. Cell type coupling factors were calculated as measures of functional connectivity to build network graphs of interactions between cell types. c, Task-specific networks generated by selecting for neurons with significant encoding for a given task factor in the task GLM. Networks are sorted according to average edge strength. d, Network strength across task networks. Dotted line corresponds to strength of shuffled network. e, Strength and variability of functional connectivity in network edges across task networks. Network edges with significantly high strength and low variability are indicated with a box $(P<0.05$, permutation test). Shaded region in a corresponds to s.e.m. $n=1996$ neurons, Direction; 1374 neurons, SampleEARly DELAY; 1076 neurons Samplelate delay; 360 neurons, Category; 623 neurons, Choicetest; 830 neurons, Choicereport; 898 neurons, touch onset 1033 neurons, touch offset; 864 neurons, kinematics; 273 neurons, noncoding from 7 animals. 

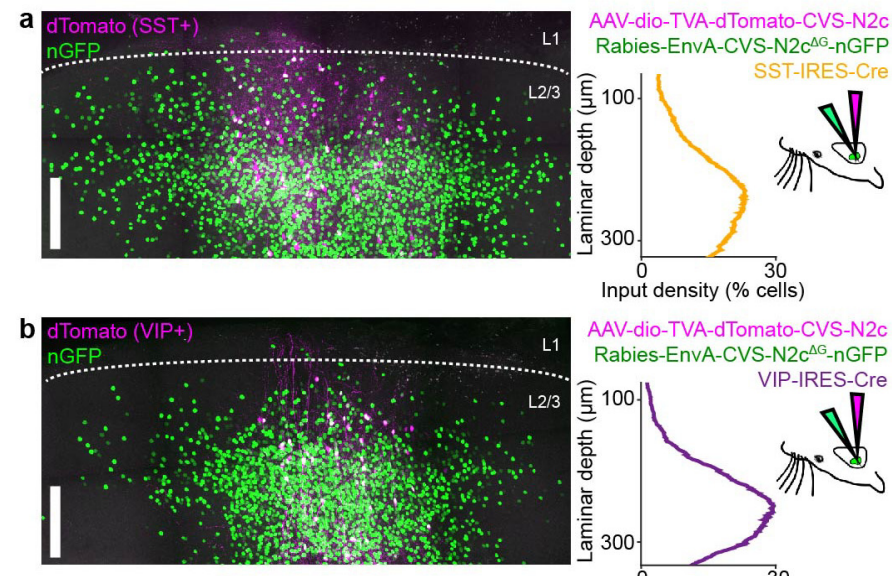

AAV-dio-TVA-dTomato-CVS-N2C

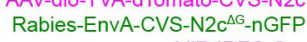
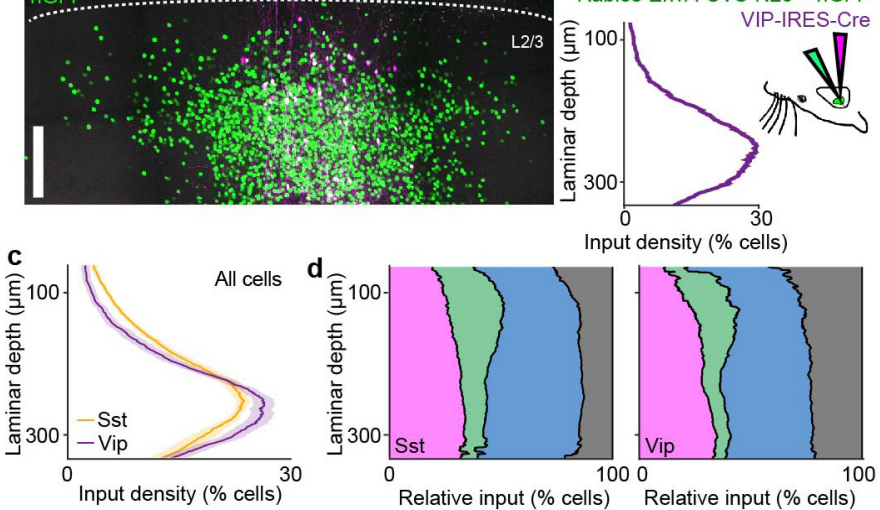

nput density (\% cells) - Adamts2 Baz1a agmat $\square$ Gad2
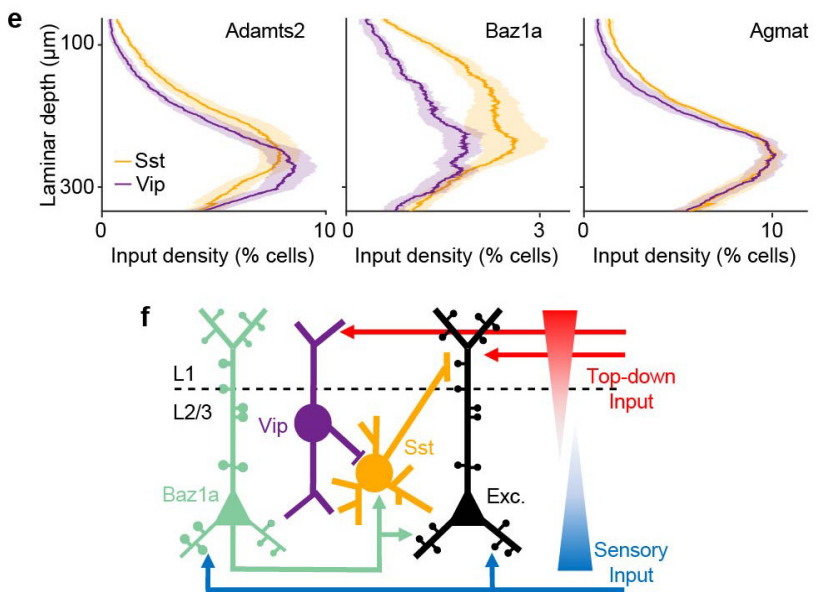

Figure 6. Upper layer Ba1za neurons target Sst neurons. a-b, Example of cell type-specific trans-monosynaptic tracing in Sst-IRES-Cre (a) and Vip-IRES-Cre (b) mice. Left panels show confocal images from coronal sections with starter cells (magenta) and nGFP+ input neurons (green). Right panels show sublaminar distribution of input density as a function of depth from left images along with injection scheme. c, Average sublaminar somatic density distribution of inputs across L2/3 for Sst and VIP neurons. d, Relative proportion of excitatory cell types and Gad2+ inhibitory neurons as a function of laminar depth for Sst and Vip input neurons. e, Sublaminar density distribution for excitatory cell types as a function of laminar depth for Sst and Vip input neurons. f, Circuit model of L2/3 illustrating cell-type-specific connectivity between Vip, Sst, Bazla, and other local excitatory neurons. Sensory-driven activation of Bazla neurons can inhibit top-down inputs via activation of Sst neurons, biasing integration of sensory and recurrent inputs in local excitatory neurons. Shaded region in c,e corresponds to s.e.m. $n=16$ slices, 33,957 neurons from 4 Sst-IRES-Cre animals, 14 slices; 35,926 neurons 4 Vip-IRES-Cre animals, 14 slices. Scale bars: $100 \mu \mathrm{m}$. 


\section{REFERENCES}

1 Tasic, B. et al. Shared and distinct transcriptomic cell types across neocortical areas. Nature 563, 72-78, doi:10.1038/s41586-018-0654-5 (2018).

2 Zeisel, A. et al. Molecular Architecture of the Mouse Nervous System. Cell 174, 999-1014 e1022, doi:10.1016/j.cell.2018.06.021 (2018).

$3 \quad$ Klingler, E. et al. A Translaminar Genetic Logic for the Circuit Identity of Intracortically Projecting Neurons. Curr Biol 29, 332-339 e335, doi:10.1016/j.cub.2018.11.071 (2019).

4 Greig, L. C., Woodworth, M. B., Galazo, M. J., Padmanabhan, H. \& Macklis, J. D. Molecular logic of neocortical projection neuron specification, development and diversity. Nat Rev Neurosci 14, 755769, doi:10.1038/nrn3586 (2013).

5 Lim, L., Mi, D., Llorca, A. \& Marin, O. Development and Functional Diversification of Cortical Interneurons. Neuron 100, 294-313, doi:10.1016/j.neuron.2018.10.009 (2018).

6 Sorensen, S. A. et al. Correlated gene expression and target specificity demonstrate excitatory projection neuron diversity. Cereb Cortex 25, 433-449, doi:10.1093/cercor/bht243 (2015).

7 Paul, A. et al. Transcriptional Architecture of Synaptic Communication Delineates GABAergic Neuron Identity. Cell 171, 522-539.e520, doi:10.1016/j.cell.2017.08.032 (2017).

8 Kepecs, A. \& Fishell, G. Interneuron cell types are fit to function. Nature 505, 318-326, doi:10.1038/nature12983 (2014).

9 Harris, K. D. \& Shepherd, G. M. The neocortical circuit: themes and variations. Nat Neurosci 18, 170-181, doi:10.1038/nn.3917 (2015).

10 Daigle, T. L. et al. A Suite of Transgenic Driver and Reporter Mouse Lines with Enhanced BrainCell-Type Targeting and Functionality. Cell 174, 465-480 e422, doi:10.1016/j.cell.2018.06.035 (2018).

11 Khan, A. G. et al. Distinct learning-induced changes in stimulus selectivity and interactions of GABAergic interneuron classes in visual cortex. Nat Neurosci 21, 851-859, doi:10.1038/s41593018-0143-z (2018).

12 Chen, K. H., Boettiger, A. N., Moffitt, J. R., Wang, S. \& Zhuang, X. RNA imaging. Spatially resolved, highly multiplexed RNA profiling in single cells. Science 348, aaa6090, doi:10.1126/science.aaa6090 (2015).

13 Choi, H. M. T. et al. Third-generation in situ hybridization chain reaction: multiplexed, quantitative, sensitive, versatile, robust. Development 145, doi:10.1242/dev.165753 (2018).

14 Shah, S. et al. Single-molecule RNA detection at depth by hybridization chain reaction and tissue hydrogel embedding and clearing. Development 143, 2862-2867, doi:10.1242/dev.138560 (2016).

15 Lubeck, E., Coskun, A. F., Zhiyentayev, T., Ahmad, M. \& Cai, L. Single-cell in situ RNA profiling by sequential hybridization. Nat Methods 11, 360-361 (2014).

16 Wang, X. et al. Three-dimensional intact-tissue sequencing of single-cell transcriptional states. Science 361, doi:10.1126/science.aat5691 (2018).

17 Nicovich, P. R. et al. Multimodal cell type correspondence by intersectional mFISH in intact tissues. bioRxiv, 525451, doi:10.1101/525451 (2019).

18 Chen, J. L., Voigt, F. F., Javadzadeh, M., Krueppel, R. \& Helmchen, F. Long-Range population dynamics of anatomically defined neocortical networks. Elife 5, e14679, doi:10.7554/eLife.14679

e14679 [pii] (2016).

19 Treweek, J. B. et al. Whole-body tissue stabilization and selective extractions via tissue-hydrogel hybrids for high-resolution intact circuit mapping and phenotyping. Nat Protoc 10, 1860-1896, doi:10.1038/nprot.2015.122 (2015). 
94120 Choi, H. M. T. et al. Third-generation <em>in situ</em> hybridization chain reaction: multiplexed,
942 quantitative, sensitive, versatile, robust. Development 145, dev165753, doi:10.1242/dev.165753

943

944

945

946

947

948

949

950

951

952

953

954

955

956

957

958

959

960

961

962

963

964

965

966

967

968

969

970

971

972

973

974

975

976

977

978

979

980

981

982

983

984

985

986

987 (2018).

21 Ohkura, M., Sasaki, T., Kobayashi, C., Ikegaya, Y. \& Nakai, J. An improved genetically encoded red fluorescent Ca2+ indicator for detecting optically evoked action potentials. PLoS One 7, e39933, doi:10.1371/journal.pone.0039933 (2012).

22 Yao, Z. et al. A taxonomy of transcriptomic cell types across the isocortex and hippocampal formation. bioRxiv, 2020.2003.2030.015214, doi:10.1101/2020.03.30.015214 (2020).

23 Chen, J. L., Carta, S., Soldado-Magraner, J., Schneider, B. L. \& Helmchen, F. Behaviour-dependent recruitment of long-range projection neurons in somatosensory cortex. Nature 499, 336-340 (2013).

24 Abs, E. et al. Learning-Related Plasticity in Dendrite-Targeting Layer 1 Interneurons. Neuron 100, 684-699 e686, doi:10.1016/j.neuron.2018.09.001 (2018).

$25 \mathrm{Yu}, \mathrm{J} ., \mathrm{Hu}, \mathrm{H}$., Agmon, A. \& Svoboda, K. Recruitment of GABAergic Interneurons in the Barrel Cortex during Active Tactile Behavior. Neuron 104, 412-427 e414, doi:10.1016/j.neuron.2019.07.027 (2019).

26 Condylis, C. et al. Context-Dependent Sensory Processing across Primary and Secondary Somatosensory Cortex. Neuron 106, 515-525 e515, doi:10.1016/j.neuron.2020.02.004 (2020).

27 Pillow, J. W. et al. Spatio-temporal correlations and visual signalling in a complete neuronal population. Nature 454, 995-999, doi:10.1038/nature07140 (2008).

28 Runyan, C. A., Piasini, E., Panzeri, S. \& Harvey, C. D. Distinct timescales of population coding across cortex. Nature 548, 92-96, doi:10.1038/nature23020 (2017).

29 Yassin, L. et al. An Embedded Subnetwork of Highly Active Neurons in the Neocortex. Neuron 68, 1043-1050, doi:https://doi.org/10.1016/j.neuron.2010.11.029 (2010).

30 Jouhanneau, J.-S. et al. Cortical fosGFP Expression Reveals Broad Receptive Field Excitatory Neurons Targeted by POm. Neuron 1065-1078, doi:https://doi.org/10.1016/j.neuron.2014.10.014 (2014).

31 Yap, E. L. \& Greenberg, M. E. Activity-Regulated Transcription: Bridging the Gap between Neural Activity and Behavior. Neuron 100, 330-348, doi:10.1016/j.neuron.2018.10.013 (2018).

32 Barth, A. L., Gerkin, R. C. \& Dean, K. L. Alteration of neuronal firing properties after in vivo experience in a FosGFP transgenic mouse. J Neurosci 24, 6466-6475, doi:10.1523/JNEUROSCI.4737-03.2004 (2004).

33 Gainey, M. A. \& Feldman, D. E. Multiple shared mechanisms for homeostatic plasticity in rodent somatosensory and visual cortex. Philos Trans $R$ Soc Lond B Biol Sci 372, doi:10.1098/rstb.2016.0157 (2017).

34 Kwan, Alex C. \& Dan, Y. Dissection of Cortical Microcircuits by Single-Neuron Stimulation In Vivo. Current Biology 22, 1459-1467, doi:https://doi.org/10.1016/j.cub.2012.06.007 (2012).

35 Tasic, B. et al. Adult mouse cortical cell taxonomy revealed by single cell transcriptomics. Nat Neurosci 19, 335-346, doi:10.1038/nn.4216 (2016).

36 Tomioka, R. et al. Demonstration of long-range GABAergic connections distributed throughout the mouse neocortex. Eur J Neurosci 21, 1587-1600, doi:10.1111/j.1460-9568.2005.03989.x (2005).

$37 \mathrm{He}, \mathrm{M}$. et al. Strategies and Tools for Combinatorial Targeting of GABAergic Neurons in Mouse Cerebral Cortex. Neuron 91, 1228-1243, doi:10.1016/j.neuron.2016.08.021 (2016).

38 Gerashchenko, D. et al. Identification of a population of sleep-active cerebral cortex neurons. Proceedings of the National Academy of Sciences 105, 10227-10232, doi:10.1073/pnas.0803125105 (2008). 
Dudai, A. et al. Barrel cortex VIP/ChAT interneurons suppress sensory responses in vivo. PLoS Biol 18, e3000613, doi:10.1371/journal.pbio.3000613 (2020).

40 Prönneke, A. et al. Characterizing VIP Neurons in the Barrel Cortex of VIPcre/tdTomato Mice Reveals Layer-Specific Differences. Cerebral Cortex 25, 4854-4868, doi:10.1093/cercor/bhv202 (2015).

41 Lee, S., Kruglikov, I., Huang, Z. J., Fishell, G. \& Rudy, B. A disinhibitory circuit mediates motor integration in the somatosensory cortex. Nat Neurosci 16, 1662-1670 (2013).

42 Hioki, H. et al. Preferential inputs from cholecystokinin-positive neurons to the somatic compartment of parvalbumin-expressing neurons in the mouse primary somatosensory cortex. Brain Res 1695, 18-30, doi:10.1016/j.brainres.2018.05.029 (2018).

43 Taniguchi, $H$. et al. A resource of Cre driver lines for genetic targeting of GABAergic neurons in cerebral cortex. Neuron 71, 995-1013, doi:10.1016/j.neuron.2011.07.026 (2011).

44 Reardon, T. R. et al. Rabies Virus CVS-N2c(DeltaG) Strain Enhances Retrograde Synaptic Transfer and Neuronal Viability. Neuron 89, 711-724, doi:10.1016/j.neuron.2016.01.004 (2016).

45 Diamond, M. E., von Heimendahl, M., Knutsen, P. M., Kleinfeld, D. \& Ahissar, E. 'Where' and 'what' in the whisker sensorimotor system. Nat Rev Neurosci 9, 601-612 (2008).

$46 \mathrm{Xu}, \mathrm{N}$. L. et al. Nonlinear dendritic integration of sensory and motor input during an active sensing task. Nature 492, 247-251 (2012).

47 Doron, G. et al. Perirhinal input to neocortical layer 1 controls learning. Science 370, doi:10.1126/science.aaz3136 (2020).

48 Spruston, N. Pyramidal neurons: dendritic structure and synaptic integration. Nat Rev Neurosci 9, 206-221 (2008).

49 Wang, Y. et al. Anatomical, physiological and molecular properties of Martinotti cells in the somatosensory cortex of the juvenile rat. J Physiol 561, 65-90, doi:10.1113/jphysiol.2004.073353 (2004).

50 Williams, L. E. \& Holtmaat, A. Higher-Order Thalamocortical Inputs Gate Synaptic Long-Term Potentiation via Disinhibition. Neuron 101, 91-102 e104, doi:10.1016/j.neuron.2018.10.049 (2019).

51 Loebrich, S. \& Nedivi, E. The function of activity-regulated genes in the nervous system. Physiol Rev 89, 1079-1103, doi:10.1152/physrev.00013.2009 (2009).

52 Margolis, D. J. et al. Reorganization of cortical population activity imaged throughout long-term sensory deprivation. Nat Neurosci 15, 1539-1546 (2012).

53 Chen, J. L., Voigt, F. F., Javadzadeh, M., Krueppel, R. \& Helmchen, F. Long-range population dynamics of anatomically defined neocortical networks. Elife 5, doi:10.7554/eLife.14679 (2016).

54 Clack, N. G. et al. Automated tracking of whiskers in videos of head fixed rodents. PLoS Comput Biol 8, e1002591, doi:10.1371/journal.pcbi.1002591 (2012).

55 Hill, D. N., Curtis, J. C., Moore, J. D. \& Kleinfeld, D. Primary motor cortex reports efferent control of vibrissa motion on multiple timescales. Neuron 72, 344-356 (2011).

56 Pnevmatikakis, E. A. et al. Simultaneous Denoising, Deconvolution, and Demixing of Calcium Imaging Data. Neuron 89, 285-299, doi:10.1016/j.neuron.2015.11.037 (2016).

57 Rupprecht, P. et al. A deep learning toolbox for noise-optimized, generalized spike inference from calcium imaging data. bioRxiv (2020).

58 Bria, A. \& lannello, G. TeraStitcher - a tool for fast automatic 3D-stitching of teravoxel-sized microscopy images. BMC Bioinformatics 13, 316, doi:10.1186/1471-2105-13-316 (2012).

59 Guizar-Sicairos, M., Thurman, S. T. \& Fienup, J. R. Efficient subpixel image registration algorithms. Opt Lett 33, 156-158, doi:10.1364/ol.33.000156 (2008). 
103460 Lou, X., Kang, M., Xenopoulos, P., Munoz-Descalzo, S. \& Hadjantonakis, A. K. A rapid and efficient 2D/3D nuclear segmentation method for analysis of early mouse embryo and stem cell image data. Stem Cell Reports 2, 382-397, doi:10.1016/j.stemcr.2014.01.010 (2014).

61 Arganda-Carreras, l. et al. Trainable Weka Segmentation: a machine learning tool for microscopy pixel classification. Bioinformatics 33, 2424-2426, doi:10.1093/bioinformatics/btx180 (2017).

62 Magnusson, K. E. G. Segmentation and tracking of cells and particles in time-lapse microscopy PhD thesis, KTH Royal Institute of Technology, (2016).

104264 Park, I. M., Meister, M. L., Huk, A. C. \& Pillow, J. W. Encoding and decoding in parietal cortex during sensorimotor decision-making. Nat Neurosci 17, 1395-1403, doi:10.1038/nn.3800 (2014). 